\title{
Article \\ Reflection-Mode Ultrasound Computed Tomography Based on Wavelet Processing for High-Contrast Anatomical and Morphometric Imaging
}

\author{
Elise Doveri, Laurent Sabatier, Vincent Long and Philippe Lasaygues *(D) \\ Aix-Marseille Université, CNRS, Central Marseille, LMA, UMR 7031, 13013 Marseille, France; \\ doveri@lma.cnrs-mrs.fr (E.D.); sabatier@lma.cnrs-mrs.fr (L.S.); long@lma.cnrs-mrs.fr (V.L.) \\ * Correspondence: lasaygues@lma.cnrs-mrs.fr; Tel.: +33-(0)484-524-283
}

check for updates

Citation: Doveri, E.; Sabatier, L.;

Long, V.; Lasaygues, P.

Reflection-Mode Ultrasound

Computed Tomography Based on

Wavelet Processing for High-Contrast Anatomical and Morphometric Imaging. Appl. Sci. 2021, 11, 9368. https://doi.org/10.3390/app11209368

Academic Editors: Richard Nauber, Lars Buettner and Jürgen W. Czarske

Received: 3 September 2021

Accepted: 2 October 2021

Published: 9 October 2021

Publisher's Note: MDPI stays neutral with regard to jurisdictional claims in published maps and institutional affiliations.

Copyright: (c) 2021 by the authors. Licensee MDPI, Basel, Switzerland.

This article is an open access article distributed under the terms and conditions of the Creative Commons Attribution (CC BY) license (https:// creativecommons.org/licenses/by/ $4.0 /)$.
Featured Application: Ultrasonic imaging of lower or upper limbs of children and adolescents.

Abstract: Medical B-mode ultrasound is widely used for the examination of children's limbs, including soft tissue, muscle, and bone. However, for the accurate examination of the bone only, it is often replaced by more restrictive clinical modalities. Several authors have investigated ultrasonic imaging of bone to assess cortical thickness and/or to estimate the wave velocity through the internal structure. The present work focuses on the transverse slice imaging process using reflection-mode ultrasound computed tomography (USCT). The method is valid for imaging soft tissues with similar acoustic impedances, but in the presence of bone, the higher contrasts alter the propagation of ultrasonic waves and reduce the contrast-to-noise ratio (CNR). There is a need to change the methods used for the processing of ultrasonic signals. Our group has developed a wavelet-based coded excitation (WCE) method to process information in frequency and time. The objective of this study is to use the method to improve reflection-mode USCT, at low ultrasonic intensities, to better address organ morphometry. Experimental results on a newborn arm phantom and on an ex vivo chicken drumstick are presented, and the usefulness of this WCE-mode USCT is discussed.

Keywords: ultrasound computed tomography; wavelet-based coded excitation; morphometric imaging; medicine for children

\section{Introduction}

Medical B-mode ultrasound [1,2] has long been imposed as the first examination modality for the diagnosis of many pediatric diseases. The other modalities (X-ray and magnetic resonance imaging scanners) are associated with various, often restrictive, inconveniences (irradiation, sedation, anesthesia of patient, and parent-child accessibility). The interest in B-mode ultrasound lies in associated operational advantages-it is painless, readily available at the bedside and not expensive-favoring regular use, and thus multiplication of diagnostic and monitoring procedures without risk. The significant use of B-mode ultrasound in medical imaging is due to the main characteristic of soft biological tissues. Indeed, the acoustic impedance contrasts between tissues are relatively small, making them ideal media for implementing linear methods of ultrasonic wave propagation. The ultrasonic wave (compression wave only) propagates in an almost homogeneous and isotropic medium, the acoustic characteristics of which are very similar to those of water. Drawbacks limit B-mode ultrasound being used as a standardized and comprehensive modality:

- B-mode ultrasound displays the acoustic impedance contrast as a grayscale map that does not quantitatively match the acoustic impedance value, or any other acoustic parameter.

- The frequencies of the transmitters used in medical practice are quite high in the ultrasonic frequency range, between $2 \mathrm{MHz}$ and $20 \mathrm{MHz}$, sometimes as high as 
$50 \mathrm{MHz}$ [3]. The higher the frequency, the stronger the absorption. The propagation can be limited in depth to display deep structures, structures obscured by bones, or in obese patients.

- If the acoustic impedance changes abruptly, as when passing an interface between hypoechogenic soft tissues and hyperechogenic bone structures, the wave/medium interaction phenomena are no longer linear. The linear theories and approximations used become limited. Taking into account the physical phenomena of wave propagation, wave refraction or beam deflection, the unattenuated shear waves, or the inhomogeneity induced by high echogenicity, modifies the acquisition protocols or image reconstruction strategies requiring the resolution of nonlinear inverse problems. B-mode ultrasound is thus poorly adapted to these complex areas, raising resolution problems in the interdiaphyseal areas of joined bones (tibia/fibula, for example), leading to a lack of distinct information between the overlying tissue (periosteum) and the underlying tissue (the deep face of the cortical bone and the medullary canal).

Ultrasound computed tomography (USCT) seems to be an alternative or complementary modality to B-mode ultrasound, including mathematical modeling more adapted to the physical phenomena induced. USCT provides access to significant acoustic parameters of biological tissues. The signal acquisition geometry is no longer linear or sectoral as in B-mode ultrasound, but circular in the orthogonal plane. USCT makes it possible to combine morphometric imaging related to an interface resolution problem and quantitative imaging related to the identification of physical parameters.

In diffraction mode, USCT exploits the information resulting from the diffraction of an incident acoustic field by an organ, and more particularly the mutual information from several transducers or several elements of an ultrasonic array [4-7]. Diffraction-mode USCT is effective for imaging the soft tissues of an organ, for example a breast, for which a fluid-like model is workable, and provides promising benefits [7]. However, USCT can be hampered by the acquisition of a large volume of recorded data, long processing times, or high computation costs, especially for iterative nonlinear approaches [8-18]. The computational cost issue tends to be less limiting nowadays thanks to the continuous increase in the power of computers and high-performance computing systems, so that USCT could become, in the medium term, an interesting modality for complete organ imaging. Nevertheless, convergence issues still limit iterative approaches when the organ, for example a limb, contains bones. For a more simplified and faster application, the reflection mode can be preferred.

In reflection mode with a single transducer or single array, USCT can be viewed as a combination of B-mode ultrasound for the physical acoustic propagation, and X-ray tomography for the image construction process [19]. It is a linearized inversion of the inverse acoustic scattering problem corresponding to imaging perturbations of a known reference medium. Acquisition and processing times are respectively reduced to a few seconds ( $<2 \mathrm{~s}$ with a 128-element multiplexed antenna) and a few minutes $(<1 \mathrm{~min}$ with a desktop computer). The processing of sinograms (sets of signals depending on the angle of incidence) is based on the well-known algorithm of reconstruction by summation of filtered backprojections or backpropagation (FBP). The image is morphological and remains qualitative. Quantitative parametric approaches have been proposed by several groups, but the pre- and postprocessing times are increased [20,21]. Recently, Wiskin et al. [15,16] proposed a compound (reflection/transmission) high-frequency (3 MHz), high-energy quantitative USCT, and the results on a complete knee organ, including the skin, the muscle and the bone, were very convincing.

The choice of the center frequency of the transducers or the array is very relevant. Clinical B-mode ultrasound and USCT of soft tissues offer essentially the same resolutions, up to $100 \mu \mathrm{m}$ for shallow tissue depths $(<5 \mathrm{~cm})$ and for frequencies above $2 \mathrm{MHz}$. When imaging an organ containing bones and fibrous muscles that attenuate the ultrasonic waves, the use of lower frequencies $(<2 \mathrm{MHz}$ ) would make it possible to avoid multiple scattering phenomena, while gaining depth of penetration to allow in-depth analysis and/or imaging 
of the interdiaphyseal area. But the spatiotemporal resolution of the image is reduced. The "lost" resolution can be enhanced using efficient signal processing methods, such as signal deconvolution $[22,23]$ or pulse compression $[24,25]$. Our group has developed a procedure for multiscale wavelet decomposition of the signals allowing all available wave packets to be processed in terms of frequency and time [26]. This method, called "wavelet-based coded excitation" (WCE), can be used to determine, independently, the velocity of the ultrasonic wave and the path of the wave through the thickness of the cortical zone of a bone, geometrically assimilated to a succession of contiguous parallelepiped plates [20]. The method can also be adapted to the measurement of the apparent density of the bone mass $[25,27]$. When adapted to a circular antenna and to canonical targets, the method improves the contrast by at least $11 \%$ [28].

The clinical use of B-mode ultrasound requires the control of potentially induced biological effects, particularly when the generated power is increased to improve propagation distances. The mechanical index (MI) and the thermal index (TI), two adequate predictors of these effects, are controlled, varying from one device to another, and from one examination configuration to another; in general lower than 1.9 for MI, and 1.4 for TI for an average power of $100 \mathrm{~mW} / \mathrm{cm}^{2}$. When these limit values are respected, the effects are considered negligible [29]. Therefore, the intensity of the transmitted waves must be taken into account. It depends on the distance from the transmitter, the duration of the signals, and the repetition periods. The intensity measured in the axis of the transducer at the focal point of the acoustic field, and related to the insonification surface (spatial-peak temporal-average intensity $\left(\mathrm{I}_{\text {spta }}\right)$ ), is used to determine the threshold that must not be exceeded to avoid tissue damage [30]. Depending on the equipment and the configuration of the examination, this intensity is between 30 and $200 \mathrm{~mW} / \mathrm{cm}^{2}$.

Because the overall project involves pediatric ultrasonic imaging of the upper and lower limbs, the transmitted intensity must be low so as not to damage young or growing tissue. However, the propagation in highly attenuating tissues such as skin, muscle, and bone is altered at low intensity. In the present work, reflection-mode USCT is studied by combining three low-ultrasonic frequencies $\left(\mathrm{f}_{0}=500 \mathrm{kHz}, 1 \mathrm{MHz}\right.$ and $\left.2.25 \mathrm{MHz}\right)$, for lowintensity waves $\left(<30 \mathrm{~mW} / \mathrm{cm}^{2}\right)$, using successively the pulse-mode and the WCE-mode transmitted methods. USCT using single-frequency sinograms for the two transmitted methods is discussed, along with the reconstruction obtained by correcting the delays and summations of the combined multifrequency sinograms (delay-and-sum processing). The analyzed objects are a newborn arm phantom, with artificial blood vessels and bones, and a complete ex vivo chicken drumstick containing skin, fat, muscles, tibia, and fibula.

\section{Fundamental Principles of Reflection-Mode Ultrasound Computed Tomography Based on Wavelet Processing}

2.1. Projection-Like Geometry of Radon

Tomography is based on the principle that an object is determined by the set of its plane projections taken at different angles [31-33]. The definition of tomography is based on the Radon transform inversion theorem. There is a bijection between the determination of an object in a Euclidean space and its determination in the associated Radon space $S$ : $\mathbb{R}^{+}$x $S$ for Euclidean space $\mathbb{R}^{2}$. A function defined in Radon space is called a sinogram. A function defined in the Euclidean space is called the image, the elements of which are pixels (2D). The reconstruction of an image is the synthesis operation of the projections as a grayscale or color map. The well-known definition of the Radon transform of the function $f$, in the spatial domain, is as follows:

$$
\begin{gathered}
\mathcal{R}: L^{1}\left(\mathbb{R}^{2}\right) \rightarrow L^{1}\left(\mathbb{R}^{+} \times S\right) \\
\mathcal{R} f=g(\boldsymbol{\beta}, \theta)=\int_{\mathbb{R}^{2}} f(\overline{\boldsymbol{\alpha}}) \delta(\boldsymbol{\beta}-\overline{\boldsymbol{\alpha}} \cdot \theta) d \overline{\boldsymbol{\alpha}}
\end{gathered}
$$


where $\bar{\alpha} \in \mathbb{R}^{2}, \beta \in \mathbb{R}^{+}$, and $\theta \in S$ are the angle of diffraction or observation. Its inverse is obtained by the operation (inversion theorem):

$$
\begin{gathered}
f(\overline{\boldsymbol{\alpha}})=\frac{1}{2 \pi} \int_{S} \int_{\mathbb{R}^{+}} g(\boldsymbol{\beta}, \theta) \otimes \delta(\boldsymbol{\beta}-\overline{\boldsymbol{\alpha}} \cdot \theta) d \boldsymbol{\beta} d \theta \\
\delta(\boldsymbol{\beta})=\frac{1}{2 \pi} \int_{\mathbb{R}}|k| e^{i k \boldsymbol{\beta}} d k
\end{gathered}
$$

where $k$ is the wave number, and $\otimes$ denotes the convolution operation. Usually, the operator between the two function spaces considered is described by a kernel denoted $K$ :

$$
\begin{gathered}
K(\overline{\boldsymbol{\alpha}}, \boldsymbol{\beta}, \theta)=\delta(\boldsymbol{\beta}-\overline{\boldsymbol{\alpha}} \cdot \theta) \\
K^{-1}(\overline{\boldsymbol{\alpha}}, \boldsymbol{\beta}, \theta)=\delta(\boldsymbol{\beta}-\overline{\boldsymbol{\alpha}} \cdot \theta)
\end{gathered}
$$

The Radon transform describes the ideal case in which the information contained in the sinogram is taken independently of any frequency bias. In practice, the information (i.e., signals) is available in a limited frequency spectrum. By introducing a frequency bias $S(k)(|S|<\infty)$, independent of the direction of observation $\theta$, and generally characterized by the width of the impulse response $s(\boldsymbol{\beta})$, the finite-frequency Radon transform is defined as follows:

$$
\check{K}(\overline{\boldsymbol{\alpha}}, \boldsymbol{\beta}, \theta)=s(\boldsymbol{\beta}) \otimes \delta(\boldsymbol{\beta}-\overline{\boldsymbol{\alpha}} \cdot \theta)
$$

Its inversion involves a deconvolution operation.

$$
\check{K}^{-1}(\overline{\boldsymbol{\alpha}}, \boldsymbol{\beta}, \theta)=s^{-1}(\boldsymbol{\beta}) \otimes \delta(\boldsymbol{\beta}-\overline{\boldsymbol{\alpha}} \cdot \theta)
$$

is the inverse Radon kernel, and $s^{-1}(\boldsymbol{\beta})$ is the spatial domain deconvolution filter.

Diffraction tomography is based on the principle that an object is determined by the information given by the diffracted field under the illumination of an incident wave field. Thus, an object to be imaged is a perturbation of a reference medium in which waves propagate.

Let us first present the ideal linear scalar case in an infinite homogeneous medium. In the Euclidean space $\mathbb{R}^{2}$, the incident and object-diffracted fields are decomposed into plane waves, and identified respectively by the normal vector $\boldsymbol{n}_{0} \in S$, associated with the angle of incidence $\phi$, and the normal vector $n \in S$, associated with the angle of observation $\theta$. The diffraction transfer function of the object $\hat{g}$ relates the diffraction field to the incident field. In the frequency domain, the diffraction transfer function of a perturbation $f$ is defined as follows:

$$
\hat{\mathrm{g}}\left(k, \boldsymbol{n}_{0}, \boldsymbol{n}\right)=\int_{\mathbb{R}^{2}} f(\boldsymbol{\alpha}) e^{-i k \cdot \boldsymbol{\alpha} .\left(\boldsymbol{n}-\boldsymbol{n}_{0}\right)} d^{2} \boldsymbol{\alpha}
$$

The equivalent in the space domain is:

$$
g\left(\boldsymbol{\beta}, \boldsymbol{n}_{0}, \boldsymbol{n}\right)=\int_{\mathbb{R}^{2}} f(\boldsymbol{\alpha}) \delta\left(\boldsymbol{\beta}-\boldsymbol{\alpha} .\left(\boldsymbol{n}-\boldsymbol{n}_{0}\right)\right) d^{2} \boldsymbol{\alpha}
$$

This relationship is similar to the Radon transform as described in Equation (2). The spatial spectrum of the object $f$ is projected onto spheres of center $-k n_{0}$ and radius $k$, defined by the equation $\mathbf{k}=k\left(\boldsymbol{n}-\boldsymbol{n}_{0}\right)$. The spatial spectrum is generally divided into two parts. The first half-sphere, defined by $\boldsymbol{n} . n_{0}>0$, corresponds to transmission measurements and gives information on the lower frequencies of the spectrum. The second half-sphere, defined by $\boldsymbol{n} . \boldsymbol{n}_{0}<0$, corresponds to backscattering measurements and gives information on the high frequencies of the spectrum.

The geometric locations associated with the Radon kernels are planes of normal $\theta$ and distant from the geometric center of the image by a distance $\beta$. The inverse kernels are identified with the high-pass-filtered versions $|k|$ of the forward kernels, depending on the variable $\beta$, defined by Equation (4). Adjoint reconstruction occurs when the adjoint 
kernel $K^{*}$ is used to reconstruct a pseudo-inverse image. The inversion algorithms are based on the discrete versions of the Radon transform $[6,31,33]$. The Euclidean space $\mathbb{R}^{2}$ is discretized into pixels. The information following $\theta$ corresponds to the projections (or signals in time). Discrete versions of the function $\delta$ are obtained by defining a discrete ramp filter. In practice, the reconstruction filter introduces oscillations that disturb the image. Several methods can be used to apodize these effects [31]. In the present work, an alternative filtering approach is studied, based on an wavelet processing method and an analogy between the Radon transform and the wavelet transform [34].

\subsection{Wavelet-Based Matched-Filter for the Inverse Radon Transform}

The wavelet transform of a function, $f \in \mathbb{R}^{2}$, is given by the set of scalar products of the function and the wavelets $\Psi_{a, b}$ which are versions compressed or dilated by a factor $a$ and translated by a vector $\boldsymbol{b} \in \mathbb{R}^{2}$ of an analyzing wavelet $\Psi$ :

$$
T_{a, \boldsymbol{b}}(f)=\left\langle\Psi_{a, \boldsymbol{b}} / f\right\rangle=\int_{\mathbb{R}^{2}} a^{-2} \Psi^{*}\left(\frac{\boldsymbol{\alpha}-\boldsymbol{b}}{a}\right) f(\boldsymbol{\alpha}) d^{2} \boldsymbol{\alpha}
$$

where $\Psi^{*}$ is the transposed wavelet of $\Psi$, and the braket $\langle. /$.$\rangle denotes the scalar product.$ The Radon transform of a perturbation $f$ (Equations (2) and (10)) is the integral of $f(x)$ over a set of all straight lines in a subspace of $\mathbb{R}^{2}$, solution of $\langle\boldsymbol{d} / \boldsymbol{\alpha}=\boldsymbol{\beta}\rangle$ with $\boldsymbol{d} \in \mathbb{R}^{2}$ a vector of unit length $(|\boldsymbol{d}|=1)$ identified with the angle of observation $\theta$, with the incident $n_{0}$ and observation $\boldsymbol{n}$ directions:

$$
g\left(\boldsymbol{\beta}, \boldsymbol{n}_{0}, \boldsymbol{n}\right)=\int_{\mathbb{R}^{2}} f(\boldsymbol{\alpha}) \delta\left(\left\langle\boldsymbol{n} . \boldsymbol{n}_{0} / \boldsymbol{\alpha}\right\rangle-\boldsymbol{\beta}\right) d^{2} \boldsymbol{\alpha}
$$

Each projection in the form of straight lines (cf. Section 2.3) is obtained by rotation and dilatation/translation of the kernel $\boldsymbol{K}$. Equation (12) can thus be analyzed as a wavelet transform, with the delta distribution $\delta$ as the analyzing wavelet such that:

$$
\Psi(\boldsymbol{\alpha})=\delta\left(\boldsymbol{n}_{0} \cdot \boldsymbol{\alpha}\right)
$$

and:

$$
T_{a, \boldsymbol{b}}(f)=\left\langle\Psi_{a, \boldsymbol{b}} / f\right\rangle=\int_{\mathbb{R}^{2}} a^{-1} \delta\left(\boldsymbol{n}_{0} \cdot \boldsymbol{\alpha}-\boldsymbol{n} \boldsymbol{\alpha}\right) f(\boldsymbol{\alpha}) d^{2} \boldsymbol{\alpha}=a^{-1} g\left(\boldsymbol{\beta}, \boldsymbol{n}_{0}, \boldsymbol{n}\right)=a^{-1} \mathcal{R} f
$$

where $\boldsymbol{b}=\boldsymbol{n} . \boldsymbol{\alpha}$ [34].

In the case of backscattering measurements $\left(n . n_{0}<0\right)$, the associated function $g\left(\boldsymbol{\beta}, \boldsymbol{n}_{0}, \boldsymbol{n}\right)$ in the space domain (i.e., the impulse response of the object) is assimilated to a projection/signal in the time domain corresponding to the backpropagated waves, leading to a spatiotemporal sinogram as a function of the angle of incidence $\phi$. In practice, the recorded signals are convolution filters between the impulse response of the object $g(t, \theta, \phi)$ and that of the electro-acoustic device $r(\mathrm{t}, \theta, \phi)$. To take these filtering effects into account, and to "achieve" the impulse responses of the object, a wavelet-based codedexcitation method (WCE) has been used [26]. This method is decomposed into two steps. First, the transmitted signal is modeled as a wavelet adapted to the electro-acoustic configuration (center frequency $f_{0}$, sampling frequency $F_{e}$, number of samples $N$ ). This will be discussed in Section 3.6. Second, the spatiotemporal sinograms are filtered by wavelet functions, with $a=2^{j}$ and $b=\frac{q}{2^{j}},(j, q) \in \mathbb{Z}^{2}$ :

$$
\psi_{j, q}(t)=2^{\frac{j}{2}} \psi\left[2^{j}\left(t-\frac{q}{2^{j}} \cdot \frac{1}{F_{e}}\right)\right]
$$

The function $\psi_{j, q}(t)$ has orthonormal functions verifying the following properties:

- $\quad \psi_{j, q}(t)$ is a function of the $L^{2}$ space;

- The Fourier transform $\hat{\psi}_{j, q}(v)$, has a compact support; 
- $\quad \psi_{j, q}(t)$ has a compact support:

$$
20 . \log \left|\frac{\psi_{j, q}(t)}{\max \left(\psi_{j, q}(t)\right)}\right|<-A d B
$$

where $A$ is a coefficient left to the free choice of the user $\left(A=10^{-3}\right.$ in this work).

The algorithm is detailed in [26]. The wavelet $\psi(t)$ is the theoretical analyzing wavelet defined by S. Jaffard [35,36] and Y. Meyer [37]. Its spectral modulus reaches a maximum at $\left(\frac{2}{3} F_{e}\right)$, and has a bandwidth equal to two octaves $\left[\frac{1}{3} F_{e} ; \frac{4}{3} F_{e}\right]$.

The so-called centered $(q=0)$ analyzing wavelets $\psi_{j, 0}(t)$ are centered on the time $t_{j}^{0}=2^{j}\left(t-\frac{1}{2 F_{e}}\right)$ and on the frequency $v_{j}^{0}=\frac{2^{j+1}}{3} F_{e}$, with the bandwidth of $\left[v_{j}^{1} ; v_{j}^{2}\right]=\left[\frac{2^{j}}{3} F_{e} ; \frac{2^{j+2}}{3} F_{e}\right]$.

For a signal $s(t)$ sampled at frequency $F_{e}$ with $N$ samples, the only nonzero scales are the dilatation scales $j \leq 0$. The wavelet coefficients are defined as the decomposition of the signal on dyadic functions, which are a convolution filters between the signal $s(t)$ and the analyzing wavelets $\psi_{j, 0}(t)$ :

$$
X_{j}(\mathrm{t})=\int_{-\infty}^{\infty} \psi_{j, 0}(t) s(t) d t=\left[s(t) \otimes \psi_{j, 0}(t)\right]
$$

If the signal $s(t)$ is equal to $\psi_{J}(t)$, which is the centered analyzing wavelet at the dilatation scale $J(J \in \mathbb{Z})$, the wavelet decomposition of this signal on the dyadic functions makes it possible to calculate the dyadic coefficients $X_{j}$ :

$$
X_{j}(t)=\psi_{J}(t) \otimes \psi_{j, 0}(t)=\left\{\begin{array}{c}
1 \text { if } j=J \text { and } q=0 \\
0 \text { elsewhere. }
\end{array}\right.
$$

The properties of the wavelet decomposition are such that the coefficients $X_{j}$ nullify everywhere except for $j=J$. This equality makes it possible to adapt the discrete high-pass filtering of the Radon inversion to a wavelet-based matched filtering of the sinogram. The inversion of the data based on the Radon transform is done. The basic principles of the projection-like Radon-geometry algorithms applied for USCT are well established for weakly variable media [7]. For imaging complete organs with hypo- and hyperechogenic structures, contrast imaging should be differentiated from obstacle imaging.

\subsection{Imaging of Hyperechogenic Media}

The investigated objects, as fields perturbations, are classified into two categories: (i) penetrable objects, corresponding to contrast imaging; and (ii) nonpenetrable objects, corresponding to obstacle imaging. Let us consider an incident plane wave excitation of pulsation $\omega$ and wave number $k=\frac{\omega}{c_{0}}$ :

$$
u(\boldsymbol{\alpha}, t)=e^{i k(\omega) \cdot \boldsymbol{\alpha}-i \omega t}
$$

where $c_{0}$ is the velocity of a wave in the known reference medium (the water in this work).

A contrast-type perturbation for penetrable objects, independent of time, is written by separating the constant part $p_{i}$ associated with the known reference medium from the contrast part $\delta p_{i}$ :

$$
p_{i}(\boldsymbol{\alpha})=p_{i}+\delta p_{i}(\boldsymbol{\alpha})
$$

The integral representation involving the Green's kernels $G$ of the problem associated with the Helmholtz operator $L$ :

$$
\begin{gathered}
u=\int_{\mathbb{R}^{2}} G L_{p} u \\
L_{p}(\boldsymbol{\alpha})=L\left(p_{i}\right)-L\left(p_{i}+\delta p_{i}(\boldsymbol{\alpha})\right)
\end{gathered}
$$




$$
L\left(p_{i}\right)=\frac{\partial L}{\partial p_{i}} p_{i}+\frac{\partial^{2} L}{\partial p_{i}^{2}} p_{i}^{2}+\ldots
$$

In addition, the diffraction transfer function $T$ of a penetrable object associated with the perturbation $\delta p_{i}$, such as soft tissues, is:

$$
T_{l i n}\left(k, n_{0}, \boldsymbol{n}\right)=\int_{\mathbb{R}^{2}} \int_{\mathbb{R}^{2}} e^{-i k n \cdot \boldsymbol{\alpha}} G\left(k, \boldsymbol{\alpha}^{\prime}, \boldsymbol{\alpha}\right) \delta p_{i}\left(\boldsymbol{\alpha}^{\prime}\right) \frac{\partial L}{\partial p_{i}}\left(e^{i k n_{0} \cdot \boldsymbol{\alpha}^{\prime}}\right) d \boldsymbol{\alpha}^{\prime} d \boldsymbol{\alpha}
$$

This formula corresponds to contrast imaging, also called Born imaging. Depending on the nature of the Green's kernels and the operator $\frac{\partial L}{\partial p_{i}}$, the kernel can be reduced to a Radon kernel in diffraction, given in Section 2.1 in Equation (10). The far-field assumption is generally used.

An obstacle-type perturbation is a finite volume in which the wave field is not defined. This volume is a form of hole bounded by a closed surface in the infinite reference medium. The diffraction behavior of an obstacle is different from that of a contrast. The wavefield $u$ defined outside an obstacle denoted $V \subset \mathbb{R}^{2}$ bounded by the surface $S$ is expressed by the Kirchhoff identity written in integral form:

$$
u=\int_{\mathbb{R}^{2}}(-G \nabla u \cdot \nabla f+u \nabla G . \nabla f)
$$

where $f$ is the characteristic function of the volume $V$. Depending on the boundary conditions at the surface of the object as an obstacle, the obstacle is called soft (Dirichlet condition); e.g., air gap in porous solid, or hard (Neumann condition); e.g., calcified structures in soft tissues. A distinction is thus made between the diffracted field in the case of a soft obstacle and in the case of a hard obstacle, and the two corresponding transfer function formulae in diffraction in the linear frame are written as:

$$
\begin{gathered}
T_{\text {soft,lin }}\left(k, n_{0}, \boldsymbol{n}\right)=\int_{\mathbb{R}^{2}} \int_{\mathbb{R}^{2}} e^{-i k n \cdot \boldsymbol{\alpha}} G\left(k, \boldsymbol{\alpha}^{\prime}, \boldsymbol{\alpha}\right) \nabla f\left(\boldsymbol{\alpha}^{\prime}\right) \cdot i k n_{0} e^{i k n_{0} \cdot \boldsymbol{\alpha}^{\prime}} d \boldsymbol{\alpha}^{\prime} d \boldsymbol{\alpha} \\
T_{\text {hard,lin }}\left(k, \boldsymbol{n}_{0}, \boldsymbol{n}\right)=\int_{\mathbb{R}^{2}} \int_{\mathbb{R}^{2}} e^{-i k n \cdot \boldsymbol{\alpha}} \nabla G\left(k, \boldsymbol{\alpha}^{\prime}, \boldsymbol{\alpha}\right) \cdot \nabla f\left(\boldsymbol{\alpha}^{\prime}\right) e^{i k n_{0} \alpha^{\prime}} d \boldsymbol{\alpha}^{\prime} d \boldsymbol{\alpha}
\end{gathered}
$$

The obstacle inversion problem is highly nonlinear, and it is very difficult to reconstruct the characteristic function $f$. In the case of a complete organ containing hyperechogenic bone obstacles and surrounding soft tissues (muscle, fat, skin), the Born-Kirchhoff "lowcontrast" approximation is no longer sufficient; the different tissues have widely differed acoustic characteristics. However, the linear framework can be applied under some assumptions if, for example, the obstacle is not too large compared with the wavelength (in the case of the Rayleigh-Mie regime).

This is the hypothesis made for the particular case of low frequency $(<2 \mathrm{MHz})$ and low intensity (the number of multiple reflections is small) imaging of small bone structures, such as the long poorly ossified bones of a child or bones of a chicken drumstick, as in this work. The bones are thus analyzed as weakly heterogeneous obstacles giving moderate amplitude echoes. In this context, the propagation can be approximated by straight lines within the organ including bones, and the ray-based inversion algorithms are relevant $[6,19]$. All perturbation parameters are defined according to the characteristics of the surrounding soft tissue, in which only compression waves propagate.

Under these fluid-like modeling conditions, the reflection-mode USCT provides a morphological reconstruction of the contours of the different tissues only. In reflection, with a single transmitter-receiver transducer, the vector $n_{0}$ associated with the angle of incidence $\phi$, and the vector $n$ associated with the angle of observation $\theta$, are collinear:

$$
\boldsymbol{n}=-\boldsymbol{n}_{0} \in S, k \in\left[k_{\text {min }}, k_{\text {max }}\right]
$$


Rotating the single transmitter-receiver transducer around the organ and transmitting pulses with a limited bandwidth corresponding to $\left[k_{\min }, k_{\max }\right]$ at each position results in a slice-by-slice spectral coverage of the object spectrum. In this work, the algorithm of reconstruction by summation of filtered backpropagation (FBP) [6] is used either with the ramp filter for pulse-mode USCT, and with the wavelet-matched filter for WCE-mode USCT.

\section{Sample, Experimental Devices, and Protocols}

\subsection{Samples}

Experiments were conducted to confirm the performance of reflection-mode USCT using the pulse-mode and WCE-mode methods on a newborn arm phantom (True Phantom Solutions, Windsor, CA, USA) and a complete ex vivo chicken drumstick. The phantom, initially used for ultrasound-guided training for nurses and physicians, was composed of a soft body, artificial blood vessels, and bones. The study was conducted at $\mathrm{H} 1=6 \mathrm{~cm}$ and $\mathrm{H} 2=14 \mathrm{~cm}$ from the tip of the hand (Figure 1). The phantom was made of durable and long-lasting materials. The blood vessels were prefilled with an ultrasoft rubberlike material containing blood-mimicking fluid. The bony part was a rubbery urethane-based composite material reinforced with ceramic powder. The bony part had a density similar to that of human bones, but a much lower velocity and a higher attenuation. Some properties of the materials constituting the phantom are presented in Table 1.

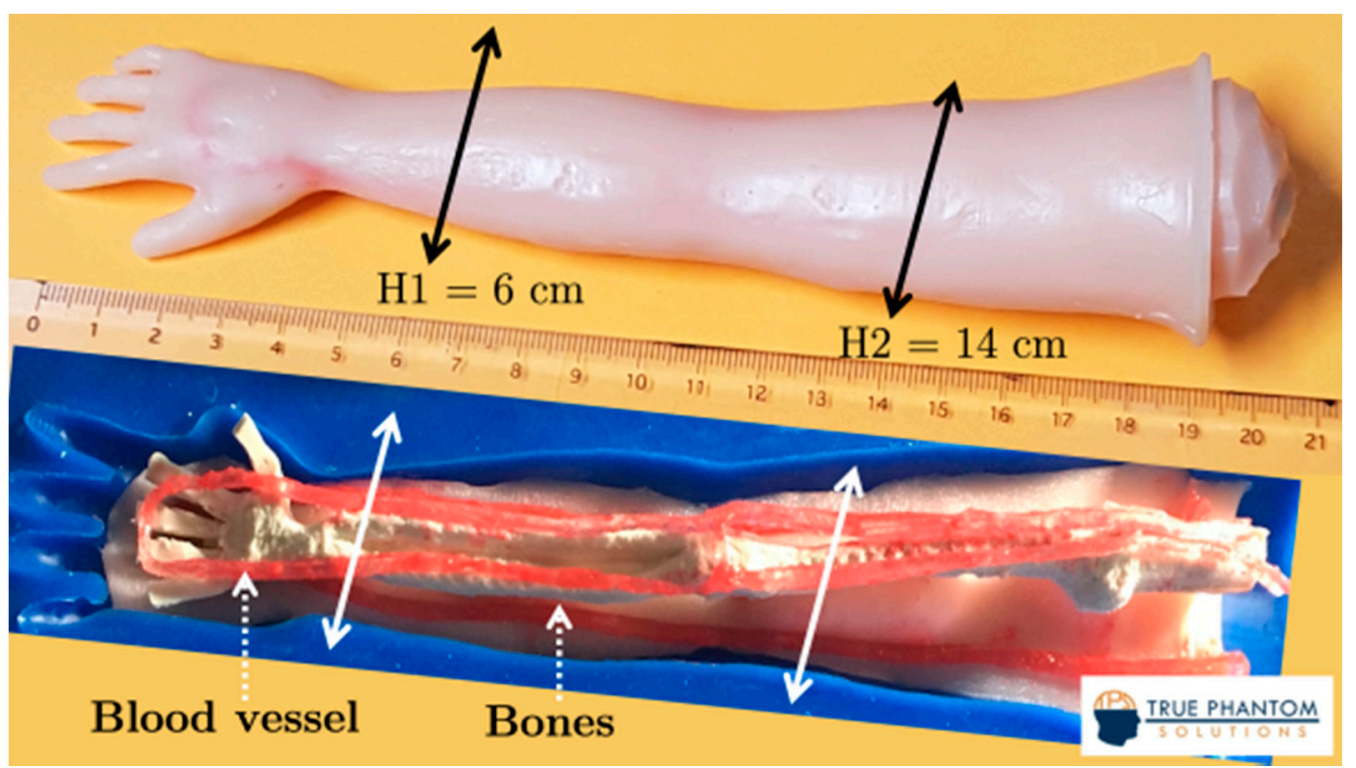

Figure 1. The newborn arm phantom (True Phantom Solutions, Windsor, CA, USA).

Table 1. Mean properties of materials constituting the newborn arm phantom (True Phantom Solutions, Windsor, CA, USA) measured at $2.25 \mathrm{MHz}$ and $1 \mathrm{MHz}$.

\begin{tabular}{ccccc}
\hline Type of Tissue & $\begin{array}{c}\text { Ultrasonic Velocity } \\
\mathbf{( m / \mathbf { s } )}\end{array}$ & $\begin{array}{c}\text { Mass Density } \\
\left(\mathbf{g} / \mathbf{c m}^{\mathbf{3}}\right)\end{array}$ & $\begin{array}{c}\text { Impedance } \\
\mathbf{( M R a y s})\end{array}$ & $\begin{array}{c}\text { Attenuation } \\
\mathbf{( d B / c m )}\end{array}$ \\
\hline Soft body & $1423 \pm 10$ & 1.0 & $\approx 1.423$ & $1.1 \pm 0.2$ \\
Blood vessel & $1400 \pm 10$ & 1.02 & $\approx 1.428$ & $1.7 \pm 0.2$ \\
Bone & $1129 \pm 5$ & 2.16 & $\approx 0.0024$ & $21 \pm 2.0$ \\
\hline
\end{tabular}

The chicken drumstick contained skin, fat, muscle, tibia, and fibula, and the study was conducted in the proximal area $25 \mathrm{~mm}$ from the upper tibio-fibular joint. The interest in this sample was that it presented well-identified adjacent structures such as muscles and skin, with nerves and fat, and had two bones, tibia and fibula, joined together. The fibula was much smaller than the tibia in the area studied in the chicken drumstick. It was 
thus possible to study the limit in resolution of USCT, and the power of detection of small bones, when low ultrasonic intensity was transmitted.

\section{2. $X-\operatorname{ray} \mu \mathrm{CT}$}

The first step of the protocol was to take reference $X$-ray images of the samples. The $\mathrm{X}$-ray tomograph used to produce the reference images was an X-ray micro-computed tomography $(\mu \mathrm{CT})$ system (EasyTom XL 150 "Mechanic Ultra", RX Solutions, Chavanod, France). The slice thickness of the X-ray cross-section was $30 \mu \mathrm{m}$ for the newborn arm phantom, and $50 \mu \mathrm{m}$ for the chicken drumstick. The number of projections was 10,080 for the newborn arm phantom, and 4320 for the chicken drumstick. The voltage of the X-ray source was $60 \mathrm{kV}$ for the newborn arm phantom, and $110 \mathrm{kV}$ for the chicken drumstick, and the beam aperture was $43^{\circ}$ for both. The image size was $1840 \times 1456$ pixels $^{2}$ (16 bits). No image processing was done to enhance the contrast. The software used to display the images was Fiji ${ }^{\circledR}$ (Image ${ }^{\circledR}$ plugins) [38].

Figures 2 and 3 show, respectively, the $\mu \mathrm{CT}$ of the newborn arm phantom and of the ex vivo chicken drumstick.

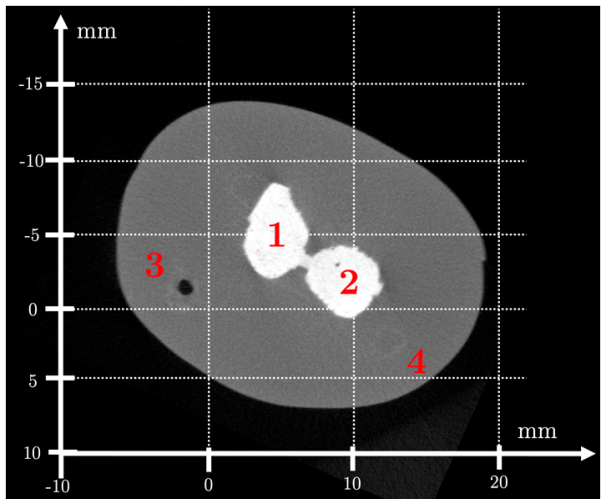

(a)

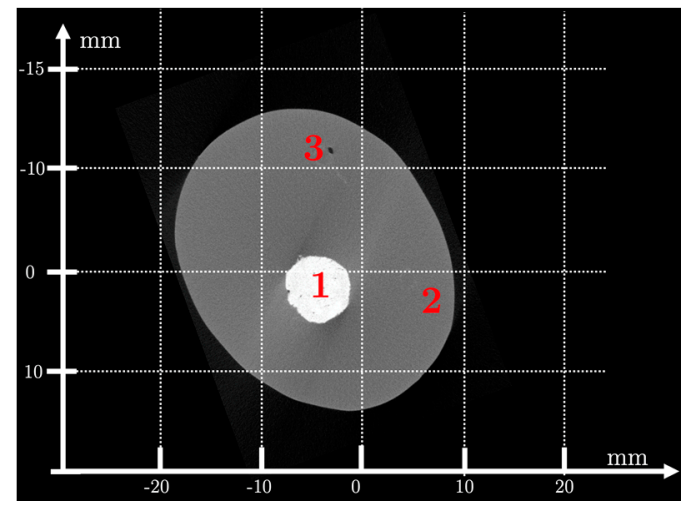

(b)

Figure 2. X-ray computed tomography $(\mu \mathrm{CT}$ ) of the newborn arm phantom (True Phantom Solutions) at $\mathrm{H} 1=6 \mathrm{~cm}(\mathbf{a})$ and $\mathrm{H} 2=14 \mathrm{~cm}(\mathbf{b})$ from the tip of the hand. Size: $1840 \times 1456$ pixels $^{2}$; resolution: $30 \mu \mathrm{m}$. Legend H1: (1-2) bones, (3-4) blood vessels. H2: (1) bones, (2-3) blood vessels.

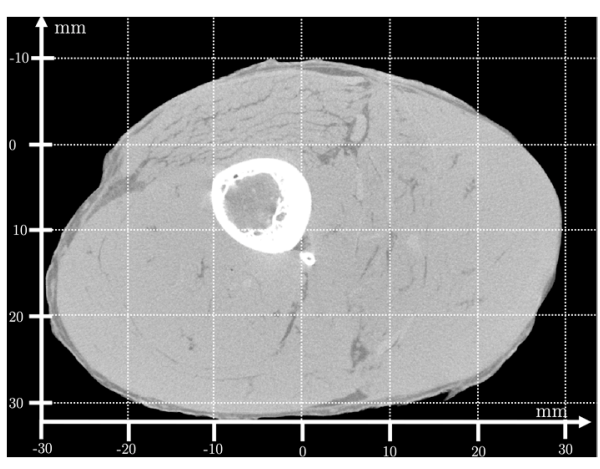

(a)

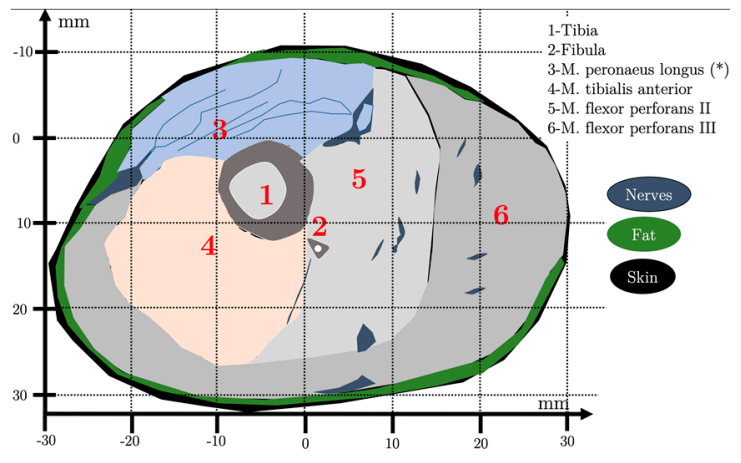

(b)

Figure 3. (a) X-ray computed tomography $(\mu \mathrm{CT})$ of an ex vivo chicken drumstick in proximal $25 \mathrm{~mm}$ to the superior tibio-fibular joint. Size: $1840 \times 1456$ pixels $^{2}$; resolution: $50 \mu \mathrm{m}$. (b) Studied region of interest and schematic topographic anatomy. Abbreviations: M. = musculus; $\left({ }^{*}\right)$ muscles fixed on the kneecap with linear long fibers.

\subsection{USCT Scanner}

Experiments were performed in reflection mode using a mechanical ultrasonic scanner consisting of a horizontal linear displacement axis, allowing correct adjustment of the 
ultrasonic beam relative to the target holder (Figure 4). As defined previously for reflectionmode USCT, the directions of incidence $\phi$ and observation $\theta$ were collinear, and the rotation of the target holder allowed angular scanning instead of rotation of the transducer around the target. In the scanning, 720 signals corresponding to 720 angles of rotation (noted $\phi$ from now on) were recorded over $360^{\circ}$, with steps of $0.5^{\circ}$. All motions were insured by stepper motors sequentially driven by a programmable translator-indexer device fitted with a power multiplexer. The translator-indexer device and power multiplexer were integrated in a control rack that also included other remote controls, such as the adjustment of the distance between targets and transducers.

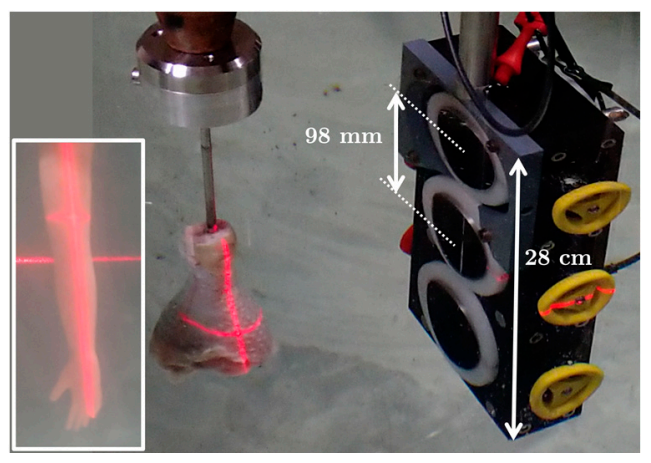

Figure 4. (Inset on the left) Newborn arm phantom. Cross-sections were chosen in areas $6 \mathrm{~mm}$ and $14 \mathrm{~mm}$ from the hand at the level of the laser cross. (Left part) Complete fresh chicken drumstick containing skin, fat, muscle, tibia, and fibula. Cross-sections were chosen in the proximal area $25 \mathrm{~mm}$ from the upper tibio-fibular joint at the level of the laser cross. (Right part) Three-frequency linear transducer array; $f_{O}=500 \mathrm{kHz}$ (bottom), $1 \mathrm{MHz}$ (middle), $2.25 \mathrm{MHz}$ (top).

\subsection{Transducers and Electro-Acoustic Devices}

The three-frequency transducers used for data acquisition were Imasonic (Voray-surl'Ognon, France) piezo-composite transducers, with center frequencies $\left(\mathrm{f}_{0}\right)$ of $500 \mathrm{kHz}$, $1 \mathrm{MHz}$, and $2.25 \mathrm{MHz}$ (Figure 4). The transducers were positioned on a $28 \mathrm{~cm}$-high linear antenna, and were $98 \mathrm{~mm}$ apart from one another. Each transducer was $60 \mathrm{~mm}$ high and $56 \mathrm{~mm}$ in outer diameter, with a lateral aperture size of $40 \mathrm{~mm}$ and an axial aperture size of $30 \mathrm{~mm}\left(12 \mathrm{~cm}^{2}\right.$, the cylindrical focalization being in the plane of the slice at $\left.150 \mathrm{~mm}\right)$, and a slice thickness of $3 \mathrm{~mm}$. It was possible to orient the acoustic beam linearly and rotationally using millimetric adjustment wheels.

The transducers were powered by two different generators for the two ultrasonic wave transmission configurations (Figure 5). The transducers were driven using a pulse-receiver generator (Sofranel 5077 PR, Olympus, Waltham, MA 02453, USA) for the pulse-mode method, and by a waveform generator (TGA 1241, Thurlby Thandar Instruments Limited, Huntingdon, UK) coupled with a diplexer (RDX-6 Diplexer, Ritec Inc., Warwick, RI, USA) for the WCE-mode method. The maximum voltage possible with the waveform generator was $10 \mathrm{~V}$ peak-to-peak. In order to have the same output power in both configurations, a power amplifier (KMP Electronic, model 44702, 150 W, 0.5-15 MHz, Sofia, Bulgaria) was connected between the waveform generator and the diplexer, and the transmitted signal dynamics were balanced between the WCE-mode and pulse-mode configurations.

The radio-frequency signals (RF-signals) were conveyed from the 12-bit oscilloscope (Lecroy HDO 6104, Teledyne Inc., Thousand Oaks, CA, USA) to a personal computer using a USB interface file transfer, and stored. Each signal contained $n=65,536$ samples, and the sampling frequency was $F_{e}=500 \mathrm{MHz}$. The signal and image processing algorithms were implemented using Matlab ${ }^{\circledR}$ (The MathWorks, Inc., Natick, MA, USA). The final size of USCT was $2048 \times 2048$ pixels $^{2}$. 


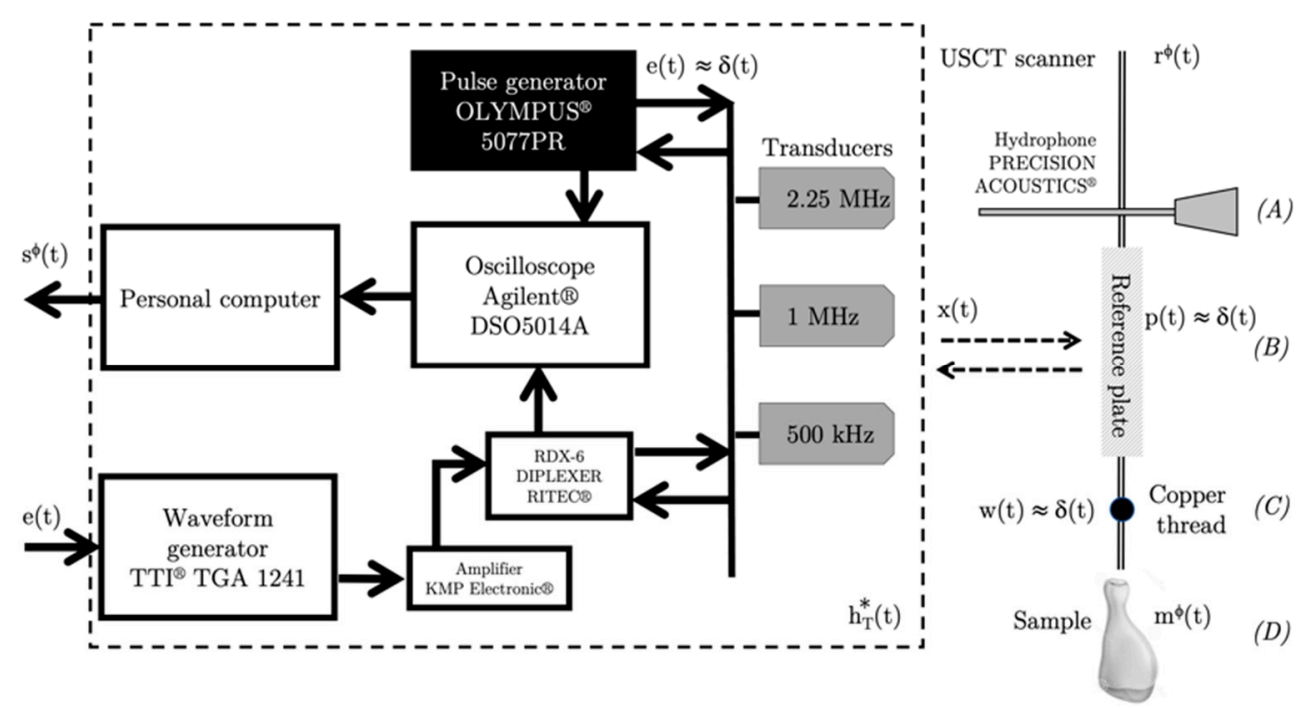

Figure 5. Synoptic view of the data acquisition and electro-acoustic setup.

Transducer calibration (bandwidth, nominal frequency, energy and intensity distribution) was carried out using a $0.5 \mathrm{~mm}$ needle hydrophone (Precision Acoustics, Dorchester, UK) mounted on the mechanical ultrasonic scanner (Figure 5, Configuration A).

A stainless-steel reference plate placed at the center of the bench (distance of $\sim 150 \mathrm{~mm}$ ) was used as a perfect reflection interface to record reference signals for the WCE-mode method. The size of the plate was more than 10 times larger than the transducer's active area (Figure 5, Configuration B).

A $70 \mu \mathrm{m}$-diameter copper thread was placed on the target holder, and was used to measure the impulse response of the mechanical apparatus (point spread function); i.e., to measure the position errors of the transducers with respect to the object during rotation (Figure 5, Configuration C).

\subsection{Experimental Conditions}

The experiment room was air conditioned and kept at a constant temperature. Experimental conditions did not change during the tests. The temperature of the water in the tank $\left(17.8^{\circ} \mathrm{C}\right)$ was determined using a digital thermometer. The ultrasonic wave velocity in the water tank $\left(c_{0}=1486 \mathrm{~m} / \mathrm{s}\right)$ was calculated using the arrival time variation measurements for two distances between the $2.25 \mathrm{MHz}$ transducer and the reference plate.

\subsection{Transmitted and Received Signals}

Figure 5 shows the synoptic view of the data acquisition and the electro-acoustic devices. The electro-acoustic devices and the transducers were analyzed as continuous linear and stationary causal filters. Let us consider an object to be imaged. Whatever the transducer used, the recorded $R F$-signal, corresponding to the response returned by the object at the rotation angle $\phi$ (i.e., the backpropagation), can be written as a function of time $t$ :

$$
s^{\Phi}(\mathrm{t})=\left(r^{\Phi} \otimes g^{\Phi}\right)(\mathrm{t}) \otimes \mathbf{x}(\mathbf{t})
$$

where $r^{\Phi}(\mathrm{t})$ is the impulse response of the mechanical apparatus including the mechanical offset of the bench depending of the angle of rotation $\phi . g^{\phi}(t)$ is the response of an object that is, according to the selected configuration, the hydrophone (Configuration $\mathrm{A}$ ); the reference plate $\left(g^{0}(\mathrm{t})=p(t)\right.$, independent of the angle of rotation $\phi$ (Configuration B); the copper thread $\left(g^{0}(t)=w(t)\right.$, independent of the angle of rotation $\phi$ (Configuration C); and the object to be imaged $\left(g^{\Phi}(\phi, t)=m^{\Phi}(t)\right)$ (Configuration D). $x(t)$ is the response of the electro-acoustic chain, and can be written:

$$
\mathrm{x}(\mathrm{t})=h_{T}^{*}(t) \otimes \mathrm{e}(\mathrm{t})
$$


$e(t)$ is the electric input conveyed to the transducer via the generators. $h_{T}^{*}$ are the impulse responses of the electro-acoustic devices, including the transmitter-receiver devices and the transducers themselves.

Pulse-mode method: USCT was first realized using an ultrasonic pulse-mode method. In this case, the electric input signal $e(t)$ was an electronic pulse signal delivered by the pulse-receiver generator, and assimilated to a Dirac delta function $(e(t) \approx \delta(t))$. The recorded $R F$-signal can then be written:

$$
\left[s^{\Phi}(\mathfrak{t})\right]^{\text {pulse }}=\left(r^{\Phi} \otimes g^{\Phi}\right)(\mathfrak{t}) \otimes h_{T}^{*}(t)
$$

The time and frequency $(-6 \mathrm{~dB})$ parameters for each transducer $\left(\mathrm{f}_{0}=500 \mathrm{kHz}, 1 \mathrm{MHz}\right.$, 2.25 MHz) are presented in Table 2. They were recorded using the hydrophone at a distance of $150 \mathrm{~mm}$ in the Fresnel zone (Configuration A). The nominal frequency was the frequency determined for the maximum amplitude of the spectrum modulus. The bandwidth was calculated for a threshold at $-6 \mathrm{~dB}$. The area under the curve (AUC) was the integral of the spectrum in the bandwidth. The pulse duration was the inverse of the bandwidth, and the axial resolution was the product of the ultrasonic wave velocity and the pulse duration.

Table 2. Time and frequency $(-6 \mathrm{~dB})$ parameters of the transmitted signal, generated using the pulse-receiver generator and recorded using a $0.5 \mathrm{~mm}$ needle hydrophone; $\mathrm{f}_{0}=500 \mathrm{kHz}, 1 \mathrm{MHz}$, $2.25 \mathrm{MHz}\left(c_{0}=1486 \mathrm{~m} / \mathrm{s}\right)$.

\begin{tabular}{|c|c|c|c|}
\hline Parameter $(-6 \mathrm{~dB})$ & $500 \mathrm{kHz}$ & $1 \mathrm{MHz}$ & $2.25 \mathrm{MHz}$ \\
\hline Nominal frequency & $477 \mathrm{kHz}$ & $858 \mathrm{kHz}$ & $2 \mathrm{MHz}$ \\
\hline Broadband width & $591 \mathrm{kHz}$ & $900 \mathrm{kHz}$ & $1 \mathrm{MHz}$ \\
\hline Bandwidth & $(229-820) \mathrm{kHz}$ & $(0.5-1.3) \mathrm{MHz}$ & (1.5-2.6) MHz \\
\hline AUC (normalized) & 0.394 & 0.459 & 0.76 \\
\hline Pulse duration $\left(t_{p u l s e}\right)$ & $1.7 \mu \mathrm{s}$ & $1.2 \mu \mathrm{s}$ & $1 \mu \mathrm{s}$ \\
\hline Axial resolution & $2.5 \mathrm{~mm}$ & $1.8 \mathrm{~mm}$ & $1.5 \mathrm{~mm}$ \\
\hline
\end{tabular}

WCE-mode method: In a second phase, the WCE-mode method was used. The method requires a calibration of the electro-acoustic response $x(t)$, which must correspond to a wavelet function $\psi_{j}(t)$, as described in Section 2.2:

$$
\mathrm{x}(\mathrm{t})=\psi_{j}(t)=h_{T}^{*}(t) \otimes \mathrm{e}(\mathrm{t})
$$

The recorded $R F$-signal can then be written:

$$
\left[S^{\Phi}(\mathfrak{t})\right]^{W C E}=\left(r^{\Phi} \otimes g^{\phi}\right)(\mathfrak{t}) \otimes \psi_{j}(t)
$$

The dilatation scale $j$ was adapted to the experimental electro-acoustic configuration for the sampling frequency $F_{e}=500 \mathrm{MHz}$, and a number of samples $N=65,536$, and was equal to -9 for $\mathrm{f}_{0}=500 \mathrm{kHz},-8$ for $\mathrm{f}_{0}=1 \mathrm{MHz}$, and -7 for $\mathrm{f}_{0}=2.25 \mathrm{MHz}$. The time and frequency (theoretical and $-6 \mathrm{~dB}$ ) parameters of the theoretical analyzing wavelets are presented in Table 3. 
Table 3. Time and frequency (theoretical and $-6 \mathrm{~dB}$ ) parameters of the theoretical analyzing wavelets. The scale $j$ was determined for each transducer with the sampling frequency $F_{e}=500 \mathrm{MHz}$, and a number of samples $N=65,536$.

\begin{tabular}{|c|c|c|c|}
\hline Theoretical Parameter & $500 \mathrm{kHz}$ & $1 \mathrm{MHz}$ & $2.25 \mathrm{MHz}$ \\
\hline$j$ & -9 & -8 & -7 \\
\hline Center frequency $\left(v_{j}^{0}\right)$ & $651 \mathrm{kHz}$ & $1.3 \mathrm{MHz}$ & $2.6 \mathrm{MHz}$ \\
\hline Bandwidth $\left[v_{j}^{1} ; v_{j}^{2}\right]$ & $(0.325-1.3) \mathrm{MHz}$ & $(0.651-2.6) \mathrm{MHz}$ & (1.3-5.2) MHz \\
\hline \multicolumn{4}{|c|}{-6 dB Parameters } \\
\hline Broadband width & $458 \mathrm{kHz}$ & $900 \mathrm{kHz}$ & $1.66 \mathrm{MHz}$ \\
\hline Bandwidth & $(248-706) \mathrm{kHz}$ & (0.5-1.4) MHz & $(1.04-2.7) \mathrm{MHz}$ \\
\hline AUC (normalized) & 0.349 & 0.694 & 1.38 \\
\hline Pulse duration $\left(t_{p u l s e}\right)$ & $2.2 \mu \mathrm{s}$ & $0.74 \mu \mathrm{s}$ & $0.5 \mu \mathrm{s}$ \\
\hline Axial resolution & $3.2 \mathrm{~mm}$ & $1.1 \mathrm{~mm}$ & $743 \mu \mathrm{m}$ \\
\hline
\end{tabular}

The challenge for the WCE-mode method was therefore to determine the signal e(t) transmitted to the generator:

$$
\mathrm{e}(\mathrm{t})=\psi_{j}(t) \frac{\otimes}{\otimes}\left[h_{T}^{*}(t)\right]^{r e f}
$$

where $\frac{\otimes}{\otimes}$ denotes the operation of deconvolution. The reference signal $\left[h_{T}^{*}(t)\right]^{\text {ref }}$ was obtained by reflection of a pulse wave on the reference plate (Figure 5, Configuration B), the impulse response of which was assimilated to a Dirac delta function $(p(t) \approx \delta(t))$. A system identification procedure based on a "black-box" model detailed in [27] was used to determine $\mathrm{e}(\mathrm{t})$. The time and frequency $(-6 \mathrm{~dB})$ parameters for the transducers are presented in Table 4 for the WCE-mode method.

Table 4. Time and frequency $(-6 \mathrm{~dB})$ parameters of the transmitted signal, generated using the waveform generator and recorded using a $0.5 \mathrm{~mm}$ needle hydrophone; $\mathrm{f}_{0}=500 \mathrm{kHz}, 1 \mathrm{MHz}, 2.25 \mathrm{MHz}$ $\left(c_{0}=1486 \mathrm{~m} / \mathrm{s}\right)$.

\begin{tabular}{cccc}
\hline Parameter (-6 dB) & $\mathbf{5 0 0 ~} \mathbf{~ H z}$ & $\mathbf{1 ~ M H z}$ & $\mathbf{2 . 2 5} \mathbf{~ M H z}$ \\
\hline Nominal frequency & $553 \mathrm{kHz}$ & $744 \mathrm{kHz}$ & $2.23 \mathrm{MHz}$ \\
Broadband width & $458 \mathrm{kHz}$ & $900 \mathrm{kHz}$ & $1.8 \mathrm{MHz}$ \\
Bandwidth & $(267-725) \mathrm{kHz}$ & $(0.5-1.4) \mathrm{MHz}$ & $(1.1-2.9) \mathrm{MHz}$ \\
AUC (normalized) & 0.347 & 0.655 & 1.42 \\
Pulse duration $\left(t_{\text {pulse }}\right)$ & $2.2 \mu \mathrm{s}$ & $1.2 \mu \mathrm{s}$ & $0.56 \mu \mathrm{s}$ \\
Axial resolution & $3.2 \mathrm{~mm}$ & $1.8 \mathrm{~mm}$ & $832 \mu \mathrm{m}$ \\
\hline
\end{tabular}

The simulated signal was sent to the waveform generator, and a pseudo-wavelet was transmitted. All the experiment signals reflected from the objects under study were filtered by the same wavelet function, as explained in Section 2.2 (Equation (18)). The algorithm is more detailed in [26]. Signal processing for a complete sinogram took about $1 \mathrm{~min}$.

Figures 6-8 show the comparison of the temporal representations and frequency spectra of the theoretical analyzing wavelet, the transmitted pulse signal, and the pseudowavelet for each transducer, recorded using the hydrophone at a distance of $150 \mathrm{~mm}$ in the Fresnel zone (Configuration A). 


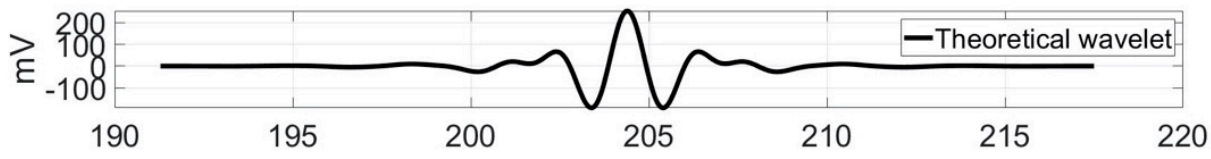

$\mu \mathrm{s}$.

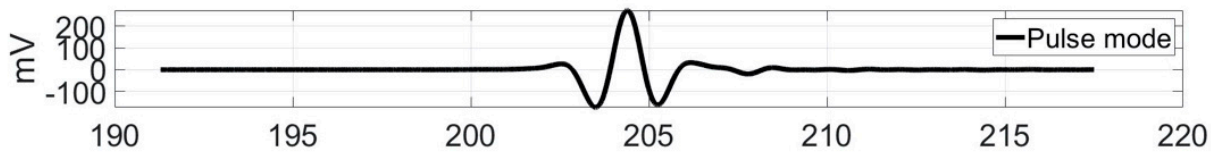

$\mu \mathrm{s}$.
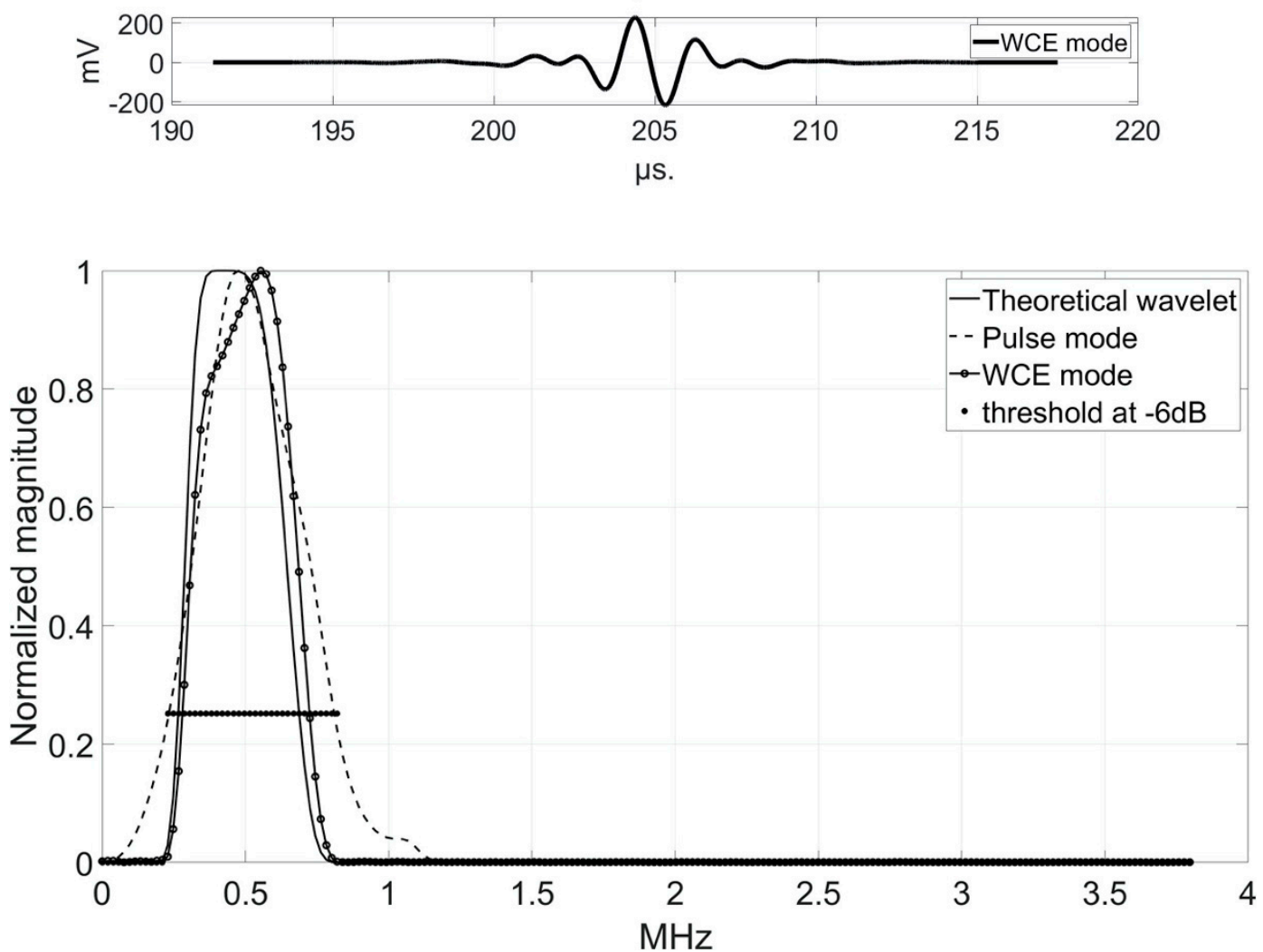

Figure 6. Comparison of the temporal representations (top) and frequency spectra (bottom) of the theoretical analyzing wavelet (solid line), the transmitted pulse signal (dashed line), and the pseudo-wavelet (dotted line) for the $500 \mathrm{kHz}-$ transducer. 


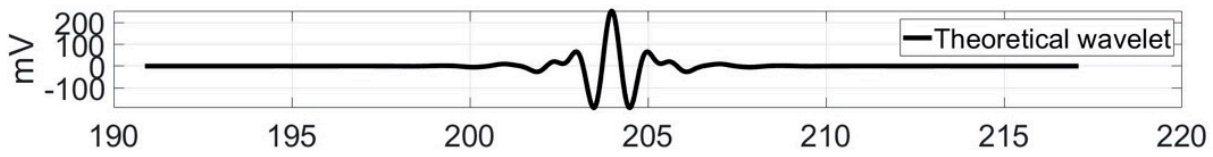

$\mu \mathrm{s}$.

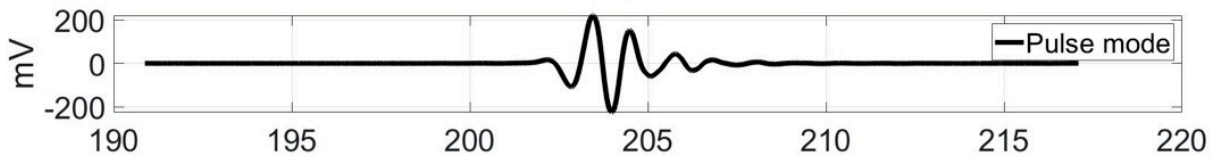

$\mu \mathrm{s}$
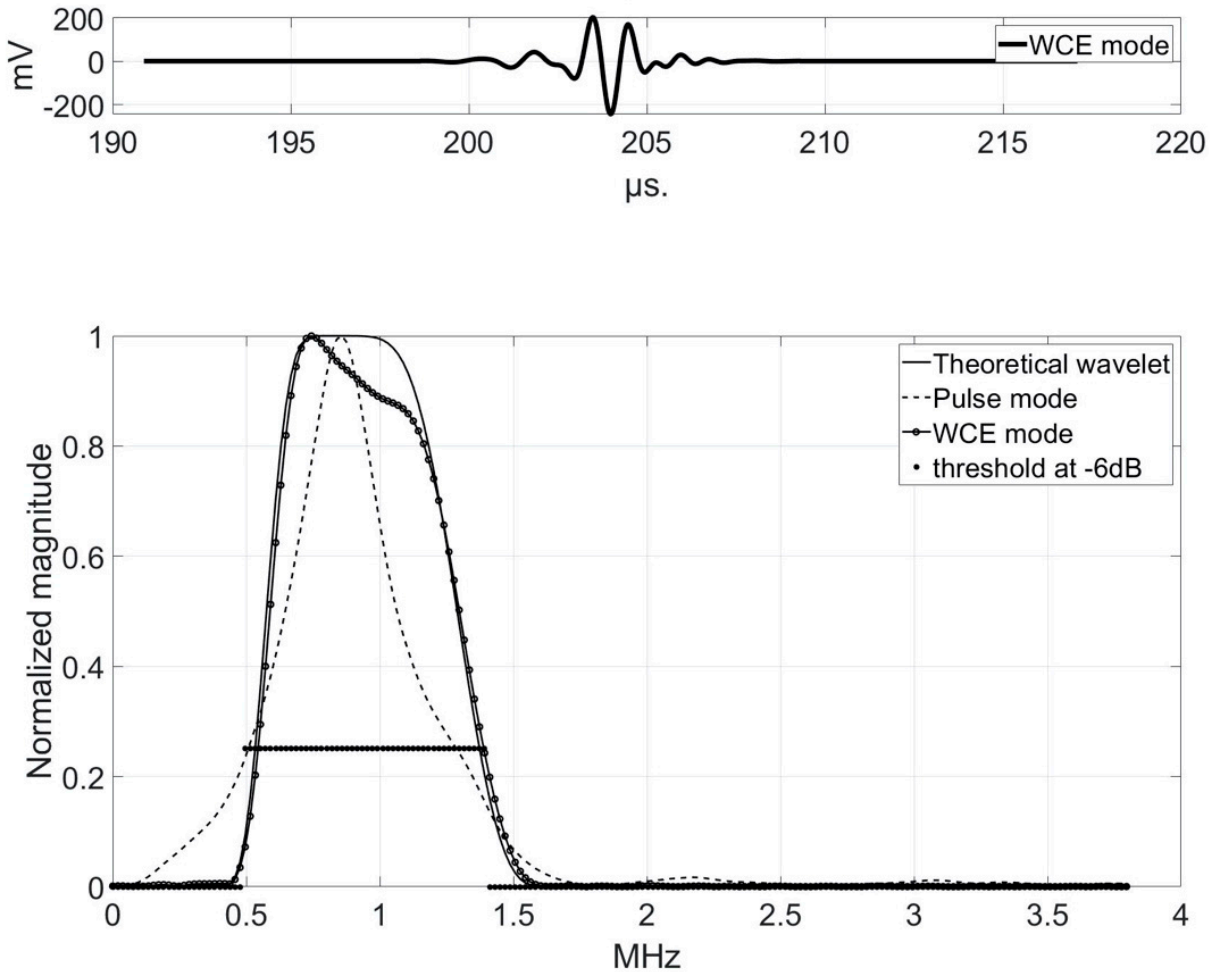

Figure 7. Comparison of the temporal representations (top) and frequency spectra (bottom) of the theoretical analyzing wavelet (solid line), the transmitted pulse signal (dashed line), and the pseudo-wavelet (dotted line) for the $1 \mathrm{MHz}-$ transducer.

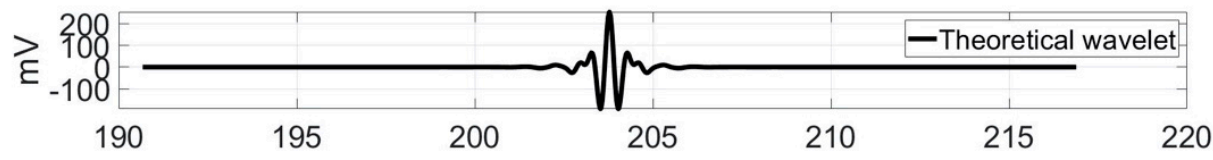

$\mu \mathrm{s}$.

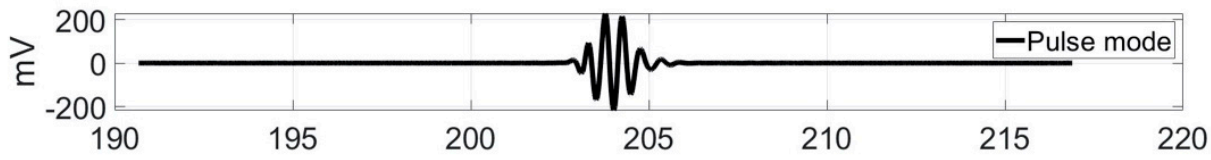

$\mu \mathrm{s}$.

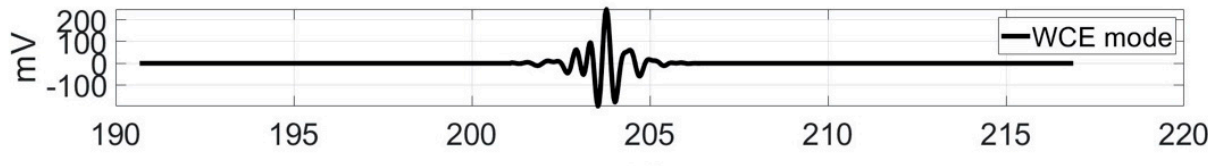

$\mu \mathrm{s}$.

Figure 8. Cont. 


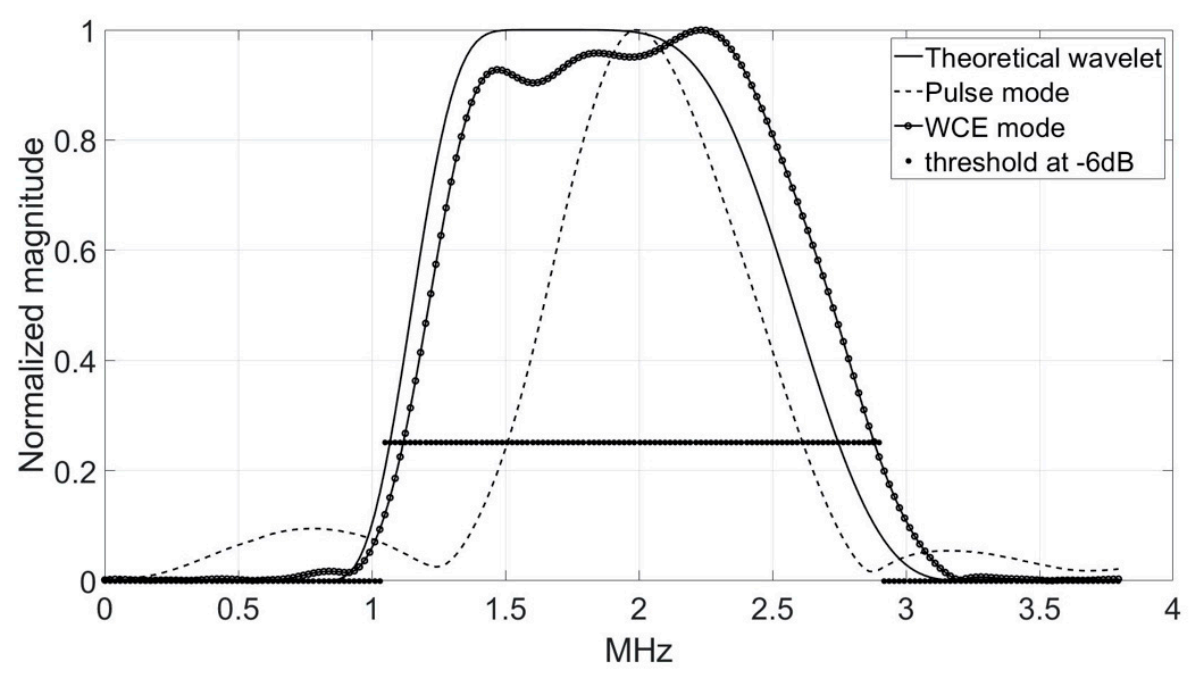

Figure 8. Comparison of the temporal representations (top) and frequency spectra (bottom) of the theoretical analyzing wavelet (solid line), the transmitted pulse signal (dashed line), and the pseudo-wavelet (dotted line) for the $2.25 \mathrm{MHz}$-transducer.

\subsection{Delay-and-Sum Processing (D.a.S.)}

Fluctuations in the position of the transducers during the rotation can give rise to time-shift variations that degrade the spatial resolution of USCT. To assess these biases, a sinogram was acquired in the pulse-mode configuration on the copper thread at the same height of the sample. The impulse response of the thin thread had a short duration, and could be assimilated to a geometric Dirac delta function. The corresponding USCT was considered as the point spread function (PSF) of the device:

$$
w(t)=\delta(t) \Rightarrow\left[s^{\Phi}(\mathbf{t})\right]^{p s f}=r^{\Phi}(\mathbf{t}) \otimes\left[h_{T}^{*}(t)\right]^{r e f}
$$

For each signal of each sinogram corresponding to each frequency obtained on the object to be imaged, the correction process consisted of a time shift in relation to the reference signal obtained at the same angle on the thread.

Once this operation was completed, the three signals (one per frequency) were summed for the same angle, and a new sinogram was compounded by delay-and-sum processing (Figure 9). An apodization coefficient $a\left(\mathrm{f}_{0}\right)$ was applied to each signal to correct the difference between the sensibilities of the three transducers, and the phase shifts $\left(\tau_{\phi}^{\left(\mathrm{f}_{0}\right)}\right)$ due to the misalignment between the transducers on the array were corrected. The misalignment was measured by reflection on the reference plate (Figure 5, Configuration B) determining the times corresponding to the maximums of the signal.

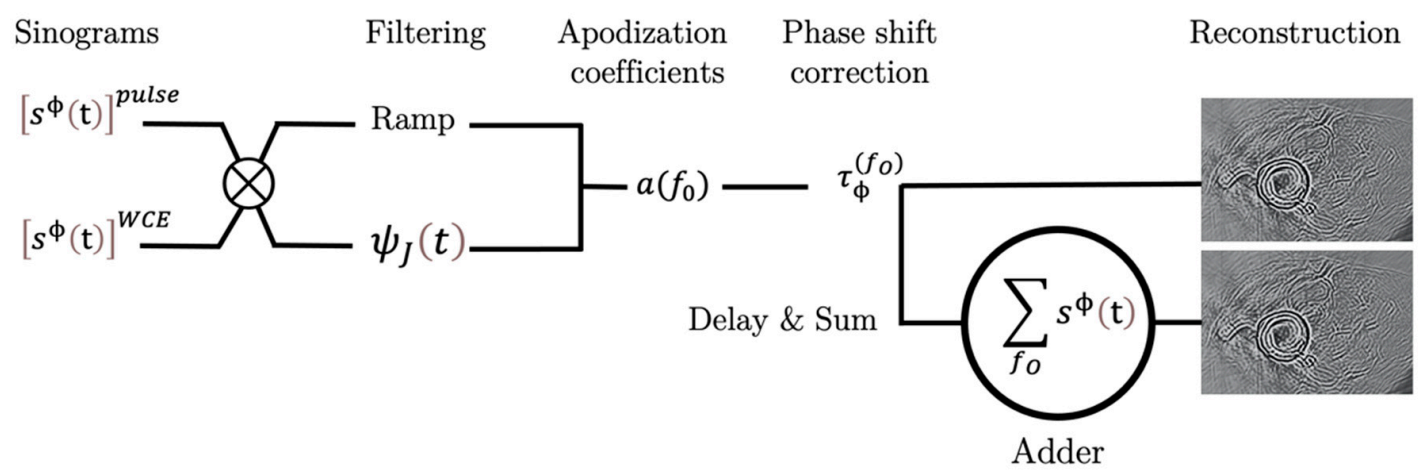

Figure 9. A flow chart of the USCT processing. 


\subsection{Acoustical Intensities}

The methods for assessing acoustic intensities were proposed by R.C. Preston [30]. There are several definitions of ultrasonic intensities, referenced by various international authorities [39], including the temporal-peak intensity $\left(I_{t p}\right)$, which describes the distribution of the sound pressure of a (plane) wave over time, and which is calculated as:

$$
I_{t p}=\frac{1}{2} \frac{p_{0}^{2}}{Z_{0}}=\frac{1}{2} \frac{\max \left(p_{+}, p_{-}\right)^{2}}{Z_{0}}
$$

where $Z_{0}=\rho_{0} c_{0}$ is the acoustic impedance of the reference medium (the water in this work).

The peak acoustic pressure $\left(p_{0}\right)$ is the greater of the two values between the maximum pressure $\left(p_{+}\right)$(i.e., maximum voltage of the recorded $R F$-signal) and the minimum pressure $\left(p_{-}\right)$(i.e., minimum voltage of the recorded $R F$-signal).

The temporal-average intensity $\left(I_{t a}\right)$ is the intensity $I_{t p}$ over the total time of ultrasonic insonification, equal to the ratio between the pulse duration $\left(t_{p u l s e}\right)$ and the pulse repetition period $\left(t_{P R P}\right)$, which was equal to $500 \mu \mathrm{s}$ in this work:

$$
I_{t a}=I_{t p} \cdot \frac{t_{p u l s e}}{t_{P R P}}
$$

The spatial-peak temporal-average intensity $\left(I_{\text {spta }}\right)$ is the maximum intensity of the spatial field slice at the focal length $\left(x_{f}=150 \mathrm{~mm}\right)$ of the transducer:

$$
I_{\text {spta }}\left(x_{f}\right)=\max _{y z}\left(I_{t a}\left(x_{f}\right)\right)
$$

where $y$ and $z$ are the coordinates of the points on the surface.

The mechanical index is expressed as follows:

$$
M I=\frac{\left|p_{-}\right|}{\sqrt{f_{O}}}
$$

where $f_{O}$ is the center frequency.

Table 5 groups the pressures, the average acoustic intensities $\left(I_{\text {spta }}\right)$, and the mechanical indexes (MI) measured using the hydrophone at the focal length $(150 \mathrm{~mm})$ of each transducer.

Table 5. The mean acoustical intensities and mechanical indexes at $500 \mathrm{kHz}, 1 \mathrm{MHz}$, and $2.25 \mathrm{MHz}$ measured using a $0.5 \mathrm{~mm}$ needle hydrophone at the focal length $(150 \mathrm{~mm})$ of each transducer.

\begin{tabular}{ccccc}
\hline Frequency $\left(\mathbf{f}_{0}\right)$ & $\begin{array}{c}\max \left(p_{+} p_{-}\right) \\
\mathbf{k P a}\end{array}$ & $\begin{array}{c}\left|p_{-}\right| \\
\mathbf{k P a}\end{array}$ & $\begin{array}{c}\boldsymbol{I}_{\text {spta }} \\
{\left[\mathbf{m W} / \mathbf{c m}^{2}\right]}\end{array}$ & $\boldsymbol{M I}$ \\
\hline $500 \mathrm{kHz}$ & 641 & -641 & 27.6 & 0.93 \\
$1 \mathrm{MHz}$ & 529.5 & -517.4 & 18.8 & 0.56 \\
$2.25 \mathrm{MHz}$ & 540.8 & -540.8 & 19.6 & 0.38 \\
\hline
\end{tabular}

\section{Results}

\subsection{Comparison of Pulse and WCE-Mode Methods for the Newborn Arm Phantom}

Figures 10 and 11 respectively show the results obtained for height $\mathrm{H} 1$ and height $\mathrm{H} 2$ of the newborn arm phantom. 


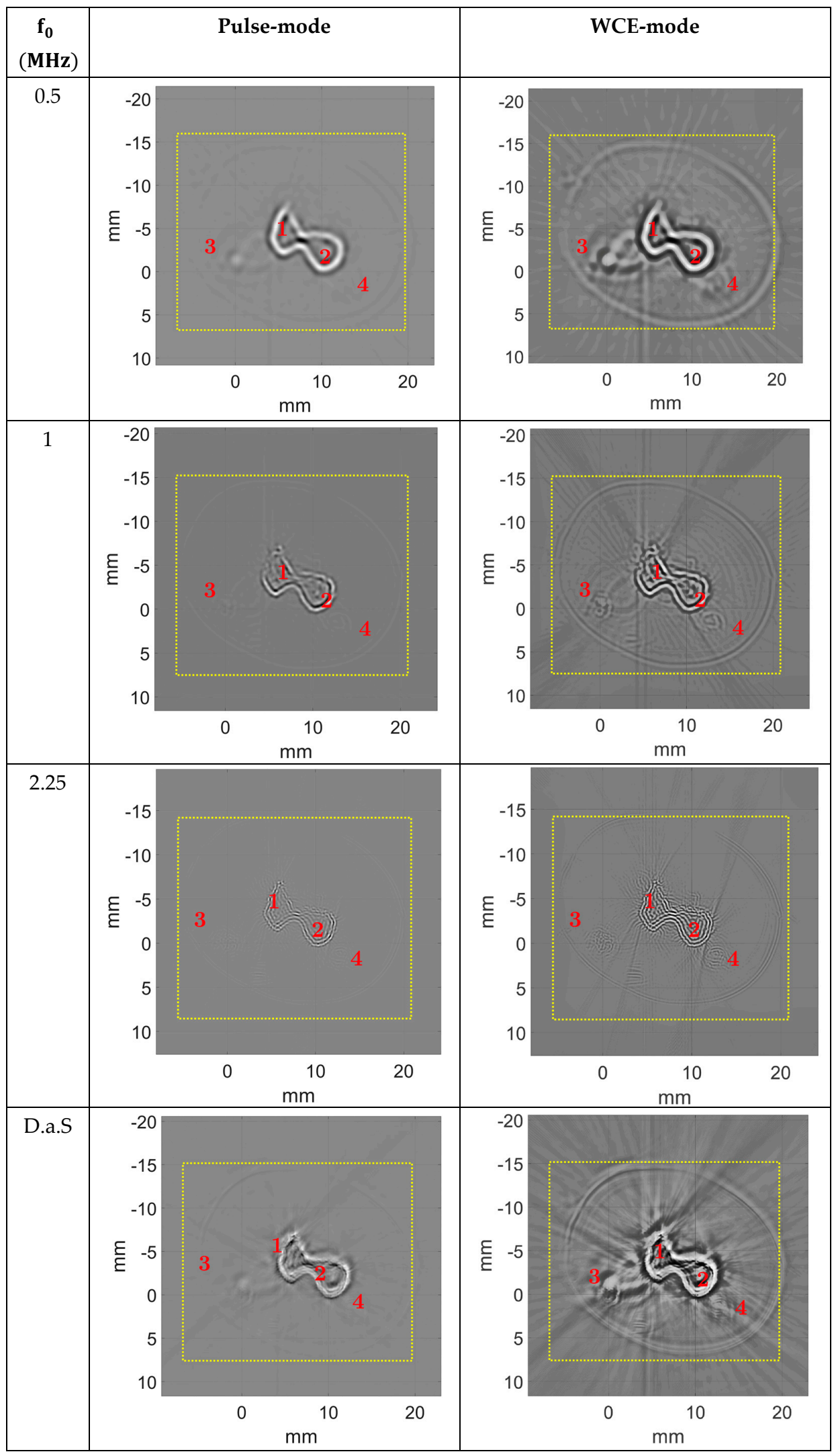

Figure 10. Reconstruction of the newborn arm phantom (True Phantom Solutions) (H1) obtained using reflection-mode USCT algorithm in pulse-mode (left) and WCE-mode (right), with 720 signals and 65,536 samples per signal. Image size: $2048 \times 2048$ pixels $^{2}$. 


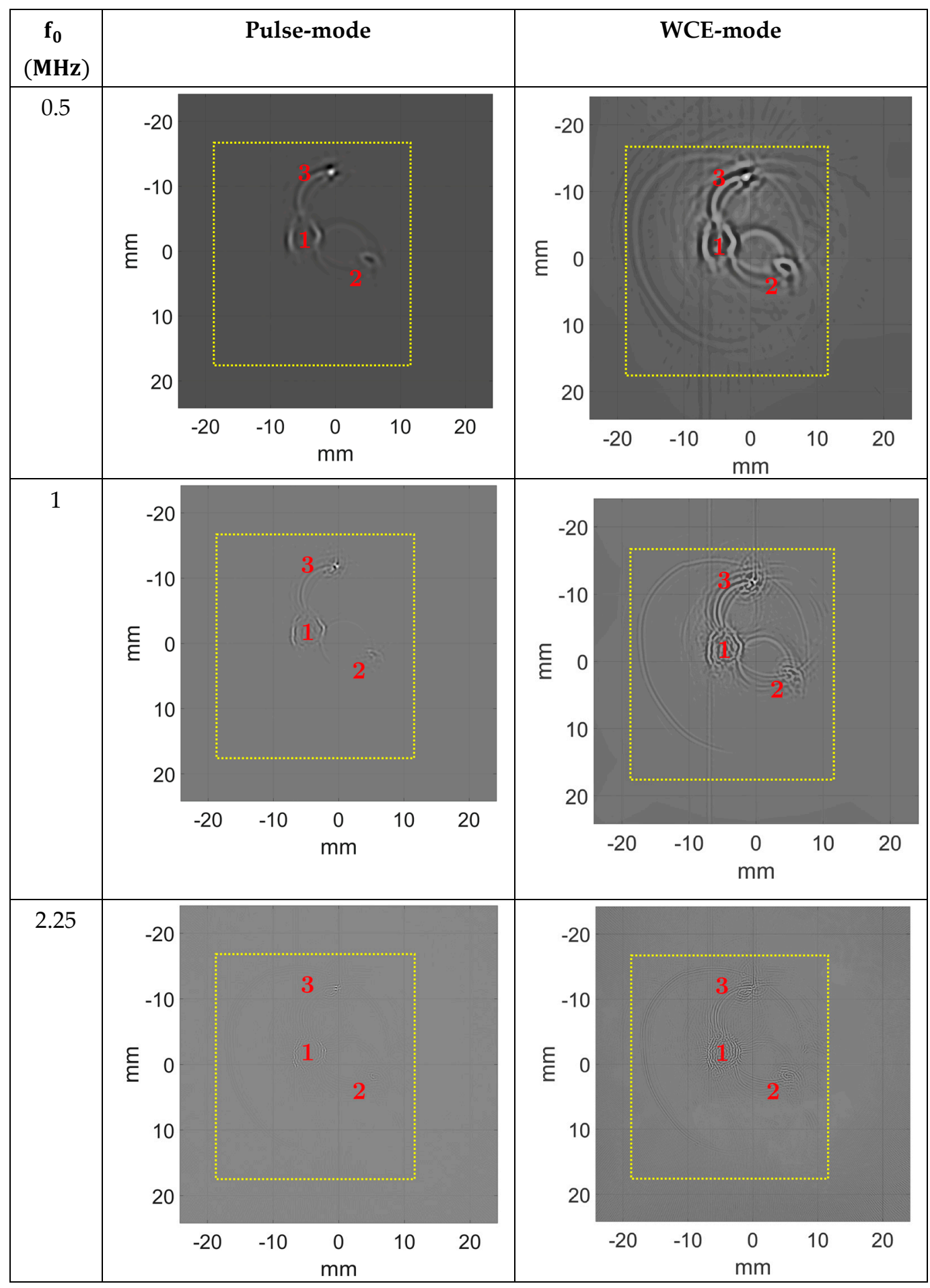

Figure 11. Cont. 


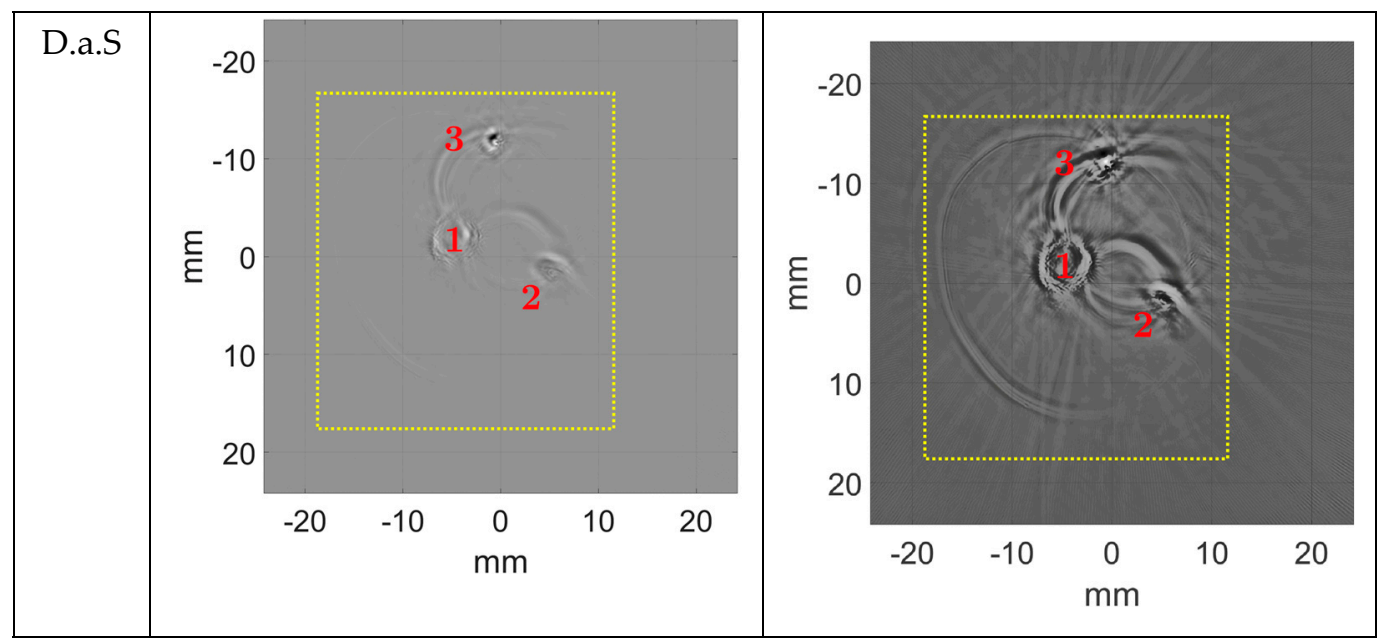

Figure 11. Reconstruction of the newborn arm phantom (True Phantom Solutions) (H2) obtained using reflection-mode USCT algorithm in pulse-mode (left) and WCE-mode (right), with 720 signals and 65,536 samples per signal. Image size: $2048 \times 2048$ pixels $^{2}$. The dotted square is the area where the contrast-to-noise ratio was calculated.

4.2. Comparison of Pulse- and WCE-Mode Methods for the Ex Vivo Chicken Drumstick Figure 12 shows the results obtained for the ex vivo chicken drumstick.

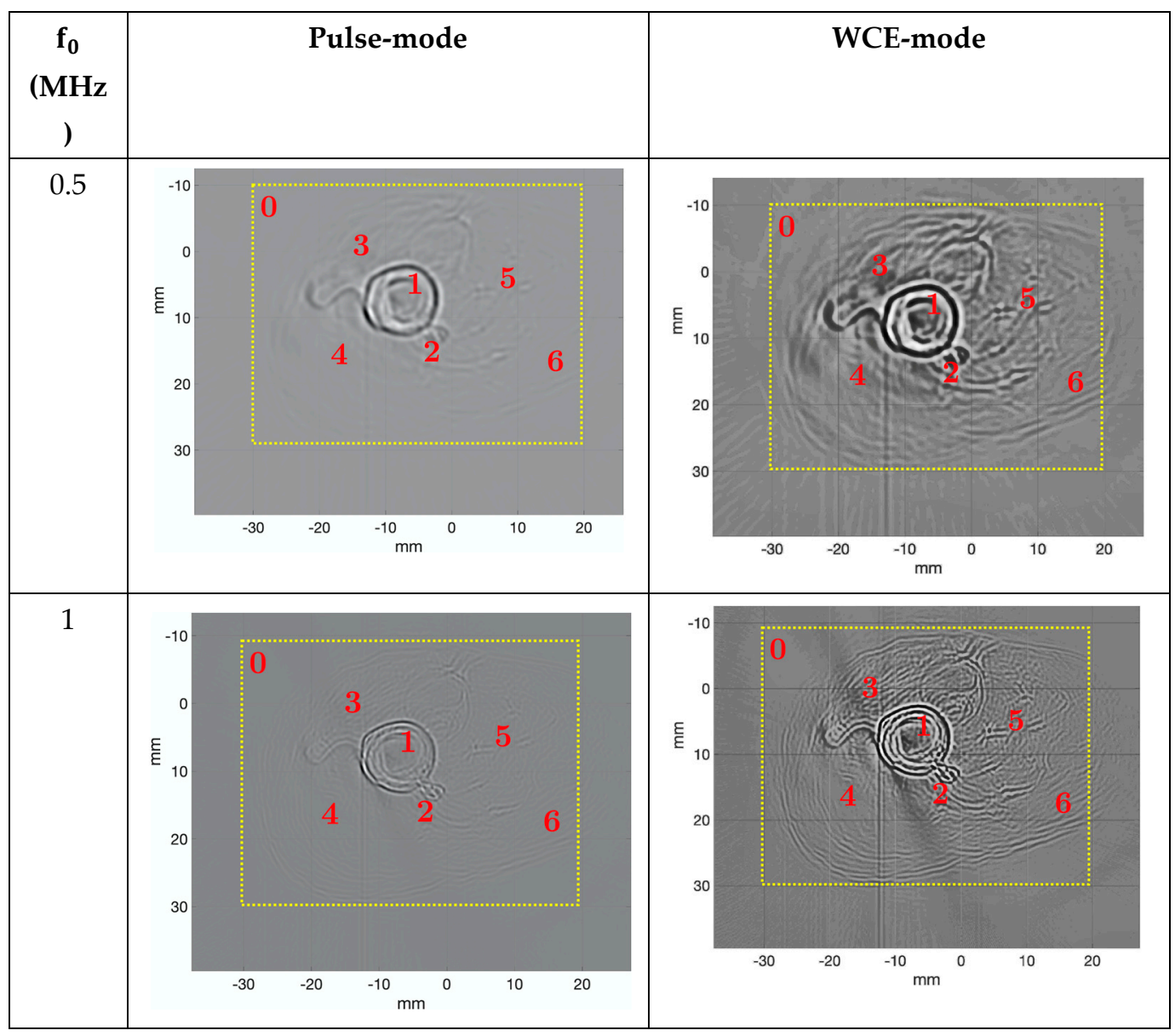

Figure 12. Cont. 


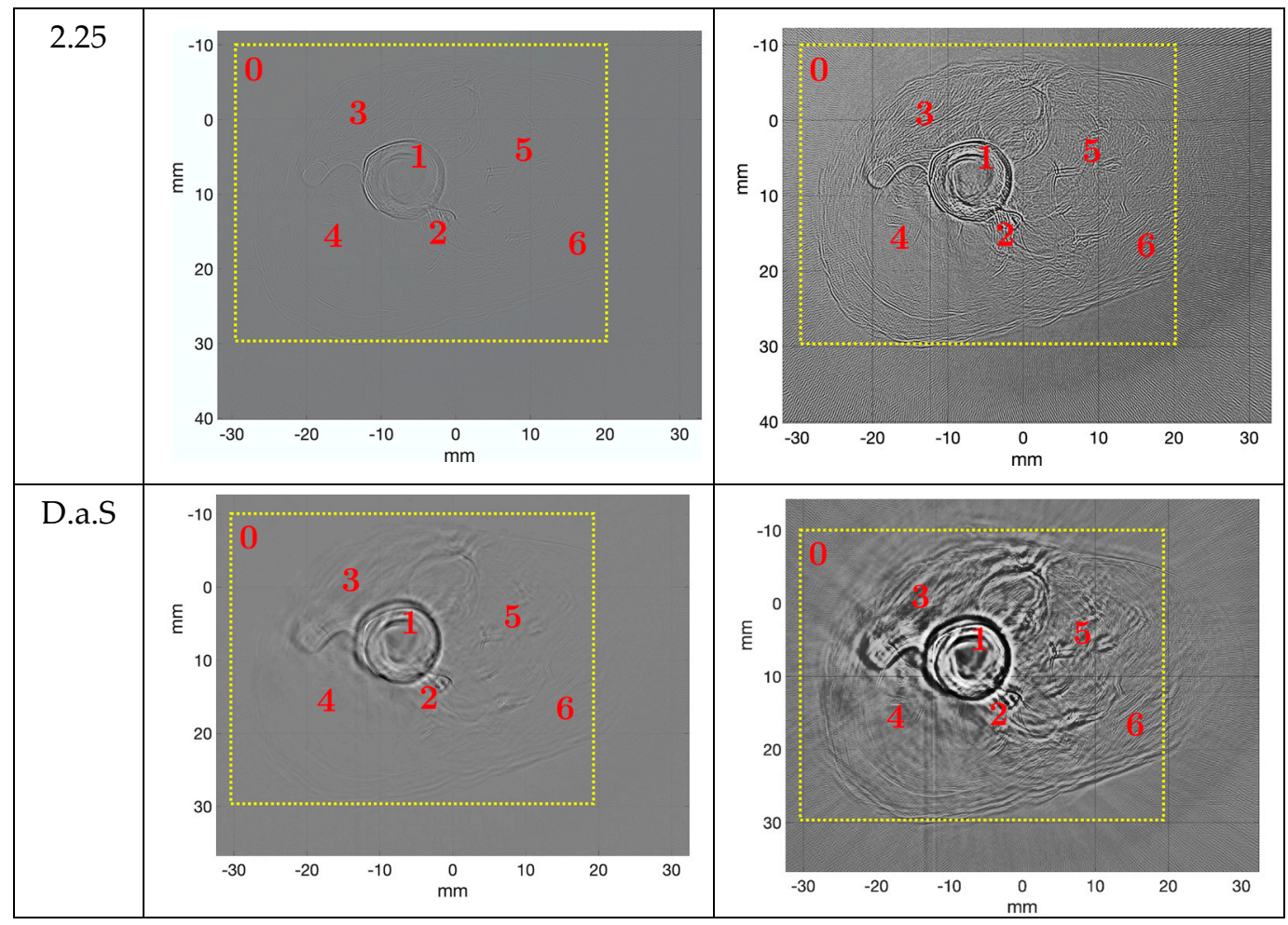

Figure 12. Reconstruction of the ex vivo chicken drumstick obtained using reflection-mode USCT algorithm in pulse-mode (left) and WCE-mode (right), with 720 signals and 65,536 samples per signal. Image size: $2048 \times 2048$ pixels $^{2}$. The dotted square is the area where the contrast-to-noise ratio was calculated.

\subsection{Study of the Contrast-to-Noise Ratio}

The contrast-to-noise ratio is defined as follows:

$$
C N R_{0, r}=\frac{\left|\overline{D_{r}}-\overline{D_{0}}\right|}{\sqrt{\sigma_{r}^{2}+\sigma_{0}^{2}}}
$$

where $\overline{D_{r}}$ and $\overline{D_{0}}$ are the mean pixel values in two areas of different brightness in the same USCT, and $\sigma_{r}$ and $\sigma_{0}$ are the corresponding standard deviations. Both areas contain the same number of pixels ${ }^{2}(200 \times 200)$. On the images, a region of interest $(\mathrm{ROI})$ around the object was defined with the coordinates $(-20,-15) \mathrm{mm}$ and $(10,15) \mathrm{mm}$ for the newborn arm phantom (Figures 10 and 11, dotted squares), and $(-30,-10) \mathrm{mm}$ and $(20,30) \mathrm{mm}$ for the ex vivo chicken drumstick (Figure 12, dotted squares). The ROIs were divided into $\mathrm{r}$ areas $\left(68 \times 68\right.$ pixels $\left.^{2}\right)$, which were assessed in comparison with the reference medium in the first area (0). As CNR increases, noise decreases and quality increases. The gain is defined in percentage by comparing the CNR obtained by the two methods:

$$
\text { GaiN }=100 *\left\{\frac{\left\langle C N R_{0, r}^{\text {wce }}\right\rangle}{\left\langle C n R_{0, r}^{\text {pulse }}\right\rangle}-1\right\}
$$

Table 6 groups the results obtained for the objects studied in this work, the newborn arm phantom and the ex vivo chicken drumstick. 
Table 6. Contrast-to-noise ratio $\left\langle C N R_{0, r}\right\rangle$ (average and standard deviation) and gain (\%) between pulse-mode and WCE-mode methods for USCT of the newborn arm phantom (True Phantom Solutions), and of the ex vivo chicken drumstick.

\begin{tabular}{|c|c|c|c|}
\hline$f_{0}(\mathrm{MHz})$ & Pulse-Mode & WCE-Mode & Gain (\%) \\
\hline \multicolumn{4}{|c|}{ Newborn arm phantom (H1) } \\
\hline 0.5 & $0.11(0.1)$ & $1.6(1.3)$ & 1355 \\
\hline 1 & $0.083(0.076)$ & $0.62(0.54)$ & 647 \\
\hline 2.25 & $1(1)$ & $3.9(5.6)$ & 290 \\
\hline D.a.S & $0.16(0.16)$ & $0.89(0.75)$ & 456 \\
\hline \multicolumn{4}{|c|}{ Newborn arm phantom (H2) } \\
\hline 0.5 & $0.13(0.1)$ & $3.5(2.6)$ & 2592 \\
\hline 1 & $0.069(0.058)$ & $1.3(1.1)$ & 1784 \\
\hline 2.25 & $0.11(0.093)$ & $1.5(0.64)$ & 1264 \\
\hline D.a.S & $0.12(0.11)$ & $2(0.99)$ & 1567 \\
\hline \multicolumn{4}{|c|}{ Ex vivo chicken drumstick } \\
\hline 0.5 & $0.3(0.23)$ & $2.1(1.4)$ & 600 \\
\hline 1 & $0.41(0.31)$ & $1.1(1)$ & 175 \\
\hline 2.25 & $0.19(0.11)$ & $0.37(0.19)$ & 95 \\
\hline D.a.S & $0.22(0.18)$ & $0.46(0.26)$ & 109 \\
\hline
\end{tabular}

\section{Discussions}

In this study, two ultrasonic wave transmission configurations adapted to reflectionmode USCT were compared for three frequencies ( $500 \mathrm{kHz}, 1 \mathrm{MHz}$, and $2.25 \mathrm{MHz}$ ), and for low ultrasonic intensities, on a newborn arm phantom and on an ex vivo chicken drumstick. The first method used a pulse as the transmitted signal, and the second used a Jaffard-Meyer wavelet adapted to the transducer frequency.

\subsection{Anatomy and Morphometry}

Regardless of imaging mode and frequency, USCT shows details not visible in $\mu \mathrm{CT}$. For example, for the newborn arm phantom, two blood vessels are clearly visible in USCT at points 3 and 4 for $\mathrm{H} 1$, and 2 and 3 for $\mathrm{H} 2$ (Figure 13), whereas they were less differentiated from the constant reference medium in $\mu \mathrm{CT}$ with the settings chosen for this experiment. Despite the low acoustic impedance contrasts between the artificial vessels and the soft body, with a low reflection coefficient between 0.0175 and 0.132 , USCT allowed visualization of the position of the vessels. Compared with $\mu \mathrm{CT}$, the orders of magnitude of the distances between the bones, the external body border, and the vessels were respected, and the tolerances were acceptable. For example, for the height $\mathrm{H} 1$ of the newborn arm phantom, at point 3 (respectively point 4 ) in $\mu \mathrm{CT}$, the distance (D and E) measured between the blood vessels and the external boundary of the phantom was $3.93 \mathrm{~mm}$ (respectively $5.55 \mathrm{~mm}$ ); whereas in the multifrequency USCT, this distance was $4.44 \mathrm{~mm}$ (respectively $6.91 \mathrm{~mm}$ ). The relative difference was about $13 \%$ (respectively $24.5 \%$ ). For the height H2, the distance (I) between the external boundary of the phantom and the bone was $9.85 \mathrm{~mm}$ in $\mu \mathrm{CT}$, and $10.05 \mathrm{~mm}$ in the multifrequency USCT with a relative difference of about $2.03 \%$. The measurements were more complicated for the blood vessels because the resolution at points 2 and 3 was not sufficient. For point 2, the size $(\mathrm{H})$ of the blood vessel was $2.16 \mathrm{~mm}$ in $\mu \mathrm{CT}$ and $2.2 \mathrm{~mm}$ in multifrequency USCT, with a relative difference of $1.85 \%$. For the bone, the diameter measurement (F) differed relatively between $\mu \mathrm{CT}(6.59 \mathrm{~mm})$ and multifrequency USCT $(4.92 \mathrm{~mm})$. The error was higher $(\approx 25 \%)$. The boundary of the phantom was identified, despite the low contrast between the tank water and the object $(\approx 1)$. The reflection coefficient tended to $1(\approx 0.997)$ between the artificial bones and the phantom body, which meant that all the waves were mainly reflected, and little energy was transmitted into the artificial bones. In addition, the material used was attenuating $(\approx 21 \mathrm{~dB} / \mathrm{cm})$. Therefore, the amount of energy transmitted beyond the artificial bones was low. Only the contours of the bones were visible, and the information inside the structures did not represent the true medium. The interdiaphyseal area was not well 
resolved, regardless of the chosen frequency. Nevertheless, the center-to-center distance (C) could be identified and was measured at $4.86 \mathrm{~mm}$ in $\mu \mathrm{CT}$ and $4.5 \mathrm{~mm}$ in the multifrequency USCT, for a relative difference of $7.41 \%$.
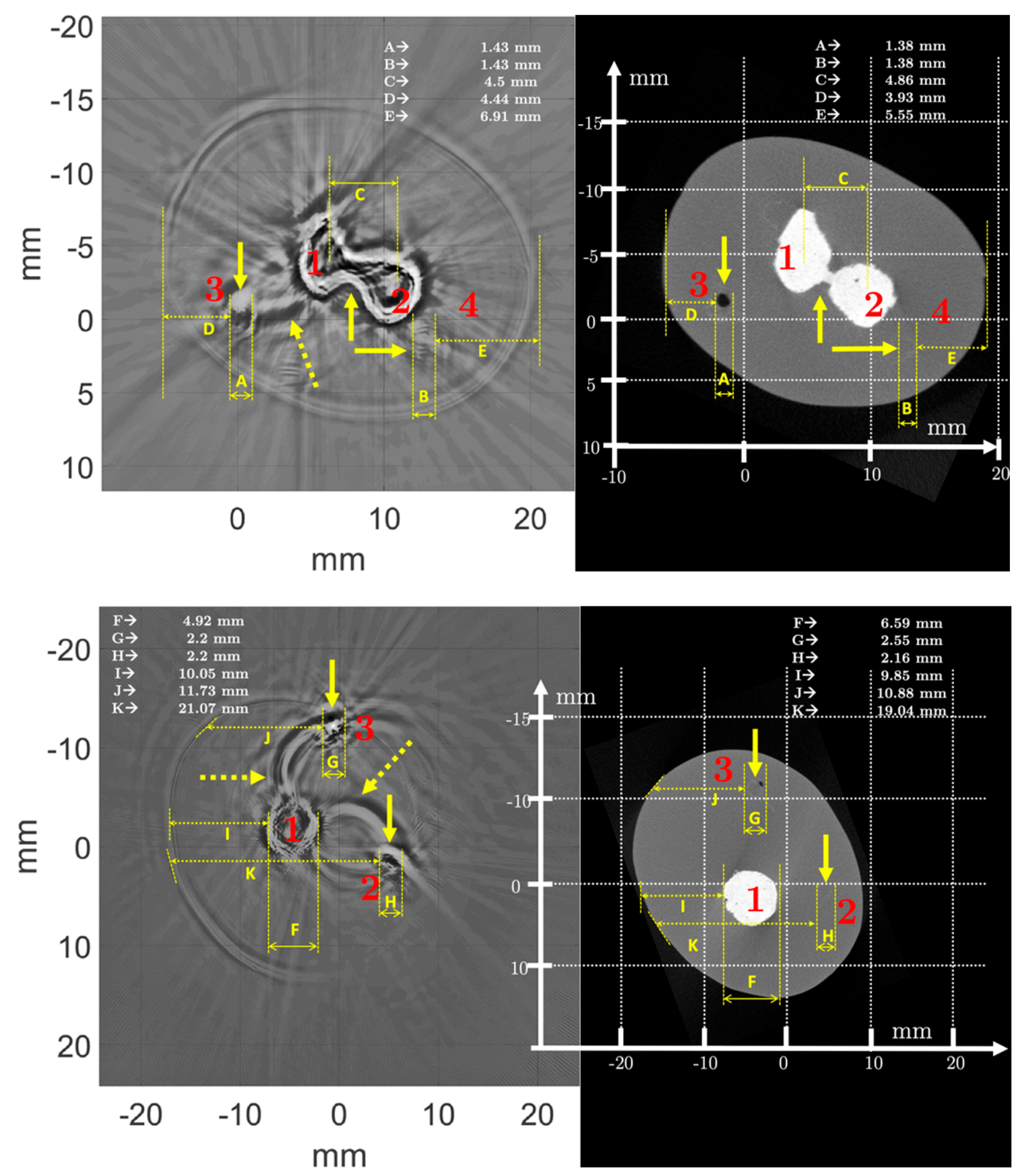

Figure 13. Morphometric analysis of the newborn arm phantom (True Phantom Solutions) by comparing USCT and $\mu \mathrm{CT}$. Solid arrows indicate areas of blood vessels and bones. Dotted arrows show artifacts.

The positioning of the calipers in the images depended on the user who was making the measurement, and was therefore imprecise and subjective, but mean relative differences of less than $10 \%$ were acceptable, especially since the low resolution of $\mu$ CT and USCT, as well as artifacts on USCT, limited the accuracy of some measurements. Indeed, in Figure 13, the dotted arrows highlight the artifacts. These artifacts interfered with the USCT analysis and did not appear in $\mu \mathrm{CT}$. They could correspond to interference waves between the artificial bones and the blood vessels. Given the high impedance contrasts between the hard and soft tissues composing the phantom, and the reflection coefficients close to 1, the waves were reflected several times between the different structures, and the reconstruction algorithm did not take these multiple echoes into account. In the $\mathrm{H} 2$ image, part of the soft body contour was not visible on the multifrequency and $500 \mathrm{kHz}$ USCT. This contour was slightly apparent on USCT at $1 \mathrm{MHz}$ and $2.25 \mathrm{MHz}$. The effects of the bone/vessel/body 
contrast were the cause of this disturbance, creating spurious signals that were difficult to eliminate.

Figure 14 shows the morphometric analysis of the ex vivo chicken drumstick, by comparison of USCT and $\mu \mathrm{CT}$. The first observation was that USCT allowed visualization, regardless of frequency and mode, of the different morphologies of the chicken thigh. The tibia (1) was very clearly visible, with a medullary part identified. The shape of the tibia was more rounded in USCT than in $\mu \mathrm{CT}$. The mean diameter (L) in USCT was greater $(11.81 \mathrm{~mm})$ than that measured in $\mu \mathrm{CT}(10.74 \mathrm{~mm})$, a relative difference of about $10 \%$. This was due to the model used for the forward and inverse problem, which assumed that all objects in the path of the waves were fluids. Since the velocity of the waves in the bone was higher than in the surrounding tissues, the waves propagating in the bone cortex, in the marrow, and then returning to the transducer arrived at a time-of-flight that did not correspond to propagation in a fluid. Even if the dimensions of the medullary canal were not realistic, USCT allowed relative measurements and identification of the different parts of the tibia. Some features of the tibia, such as the small upper protrusion (dotted circle), could be visualized. The fibula (2) was also clearly visible. The resolution in USCT was not the same as in $\mu \mathrm{CT}$, but it was quite possible to identify the fibula, distinctly from the tibia, which was much larger in the chicken drumstick. The measured diameter (M) was about $1.94 \mathrm{~mm}$ in $\mu \mathrm{CT}$ and $2.43 \mathrm{~mm}$ in USCT. The relative difference was high, and equal to about $25 \%$. The distance $(\mathrm{N})$ between the center of the tibia and the center of the fibula was about $6 \mathrm{~mm}$ in USCT, compared with $6.1 \mathrm{~mm}$ in $\mu \mathrm{CT}$. The relative difference was less than $2 \%$, which was small enough so that it could be considered that the two modalities provided close organ visualization. The use of large-aperture and broadband transducers, and the combination of several frequencies, allowed this identification. This point will be discussed. The Peronaeus longus (3), Tibialis anterior (4), and Flexor II and III (5 and 6) muscles were well identifiable. The measurements (O-P-Q-R) were respectively $24.1 \mathrm{~mm}, 19.5 \mathrm{~mm}, 13.41 \mathrm{~mm}$, and $14.07 \mathrm{~mm}$ in USCT; and $26.57 \mathrm{~mm}, 22.41 \mathrm{~mm}, 13.42 \mathrm{~mm}$, and $13.93 \mathrm{~mm}$ in $\mu \mathrm{CT}$. Even though, for point R for example, the resolution of USCT did not make it possible to be very precise, the relative differences remained lower than $13 \%$, and for some measurements lower than or equal to $1 \%$, which was encouraging. These differences were due to the reconstruction model, which considered that all the organs crossed by the compression wave were fluids, and that the wave velocity was constant and equal to that of water. The model did not take into account the variations in wave velocity and density in each of the different muscles. The highly attenuating chicken drumstick skin and nerves in areas 3,5, and 6 appeared in USCT, particularly at the right and left ends of the Peronaeus longus muscle (3). The fibers contained in this muscle were also identified.

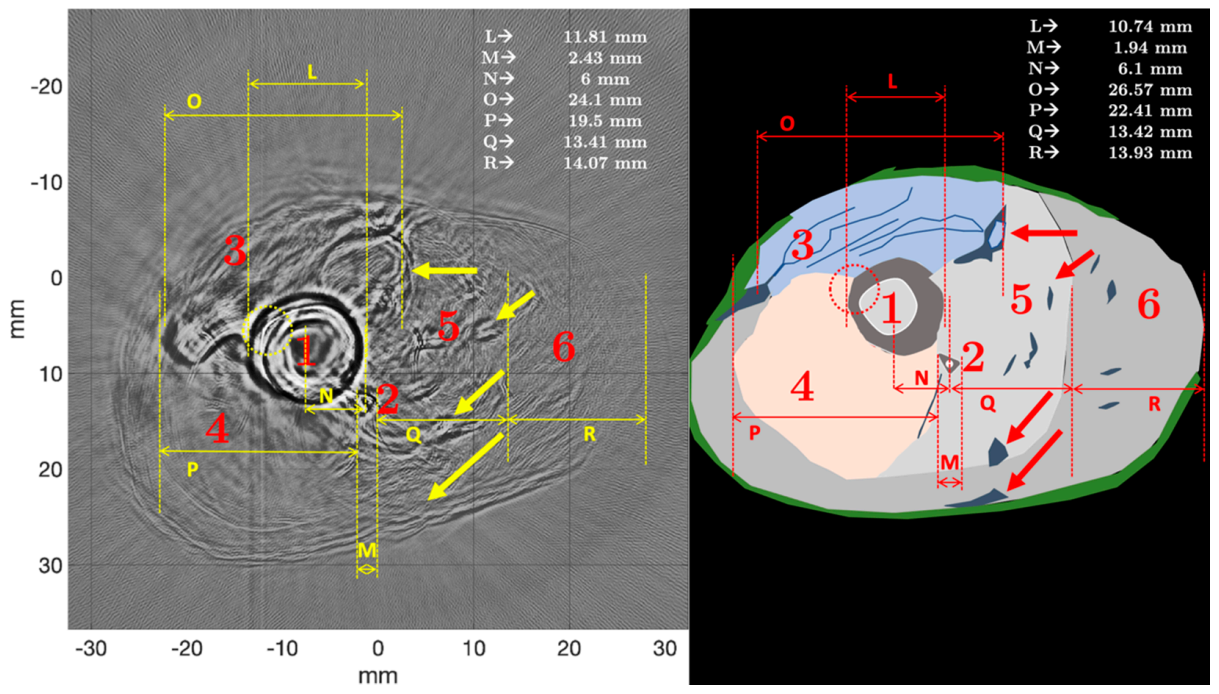

Figure 14. Morphometric analysis of the ex vivo chicken drumstick by comparing USCT and $\mu \mathrm{CT}$. The solid arrows indicate specific morphological zones. 


\subsection{Pulse-Mode USCT versus WCE-Mode USCT}

One of the objectives of this work was to compare USCT in pulse mode and in WCE mode. The study of the contrast-to-noise ratio (CNR) showed that the WCE-mode USCT was advantageous. Indeed, the gain provided by the WCE-mode method was high. It was more significant at $500 \mathrm{kHz} ;>2500 \%$ for USCT of the newborn arm, and $600 \%$ for the chicken drumstick (Table 6). At $1 \mathrm{MHz}$ and $2.25 \mathrm{MHz}$, the WCE-mode USCT improved the CNR markedly, but this improvement was different from object to object. For the newborn arm phantom, the gain at $2.25 \mathrm{MHz}$ was higher than that at $1 \mathrm{MHz}$. On average, the multifrequency modality did not provide any improvement in CNR. The study of the transducers and their acoustic fields, discussed below, will provide an explanation of this point.

The analysis of the acoustic signals could be of some help in understanding the relevance of the WCE-mode method. Figure 15 shows three signals selected at $5^{\circ}, 90^{\circ}$, and $336^{\circ}$ on the $1 \mathrm{MHz}$ sinograms, respectively in pulse-mode (solid line) and WCE-mode (dotted line), obtained for the chicken drumstick. Four main areas could be observed, for which the WCE-mode method improved resolution (AA), improved amplitude and resolution (BB), improved amplitude without affecting resolution (CC) or, conversely, reduced amplitude while maintaining resolution (DD). These examples illustrated the relevance of the WCE-mode method, which allowed weak echoes to be amplified, and resolution, and thus the CNR of USCT, to be improved.

The pseudo-wavelets were not similar to the theoretical analyzing wavelets. This had an influence on the pulse duration and on the axial resolution. At $2.25 \mathrm{MHz}$ for example, the axial resolution was lower in WCE mode $(832 \mu \mathrm{m}$, Table 4) than in pulse mode (1.5 mm, Table 2). At this frequency, small details were visible in WCE-mode USCT, and hardly or not at all in pulse-mode USCT. The small structures in the fibrous areas of the chicken drumstick were better resolved. On the newborn arm phantom, this effect was less remarkable, since the detection of blood vessels of low acoustic impedance contrast was not better at this frequency. At $1 \mathrm{MHz}$, the axial resolution was of the same order of magnitude for both methods, and equal to $1.8 \mathrm{~mm}$. The comparison of the images in pulse mode and in WCE mode showed that the different organs were visible, and that the contrast increased between the two modes. At $500 \mathrm{kHz}$, the axial resolution was worse in WCE-mode (3.2 mm, Table 4) than in pulse-mode ( $2.5 \mathrm{~mm}$, Table 2). Consequently, the images obtained in WCE-mode were less well resolved than those obtained in pulsemode, and the explanation of the benefit between the two modes was not related to the axial resolution for this frequency. This poor resolution at $500 \mathrm{kHz}$ also influenced the multifrequency USCT. If the theoretical analyzing wavelets had been modeled correctly, the axial resolution would have been further improved at high frequencies, but not at low frequencies (Table 3).

The error in the construction of the pseudo-wavelets therefore limited the axial resolution. The analysis of the bandwidths was related. The wider the bandwidth, the more energy was transmitted at low frequencies of the spectrum, and the better the resolution at high frequencies of the spectrum. The moduli of the pseudo-wavelet spectra were more consistent with the equivalent moduli of the theoretical analyzing wavelet spectra, and the bandwidths of the pseudo-wavelet and the theoretical analyzing wavelet were almost the same.

At $500 \mathrm{kHz}$, the bandwidth in pulse-mode $(591 \mathrm{kHz}$, Table 2) was higher than that in WCE-mode ( $458 \mathrm{kHz}$, Table 4) (Figure 6). If the theoretical analyzing wavelet had been well numerized, the improvement would still not have been sufficient; the bandwidth would have been the same, and the lowest significant frequency at $-6 \mathrm{~dB}$ would still have been higher than in pulse-mode ( $267 \mathrm{kHz}$ in WCE-mode against $229 \mathrm{kHz}$ in pulse-mode), and the highest significant frequency lower $(725 \mathrm{kHz}$ against $820 \mathrm{kHz})$. The bandwidth at $500 \mathrm{kHz}$ was broader for the signals in the pulse-mode method than for those in the WCE-mode method. 

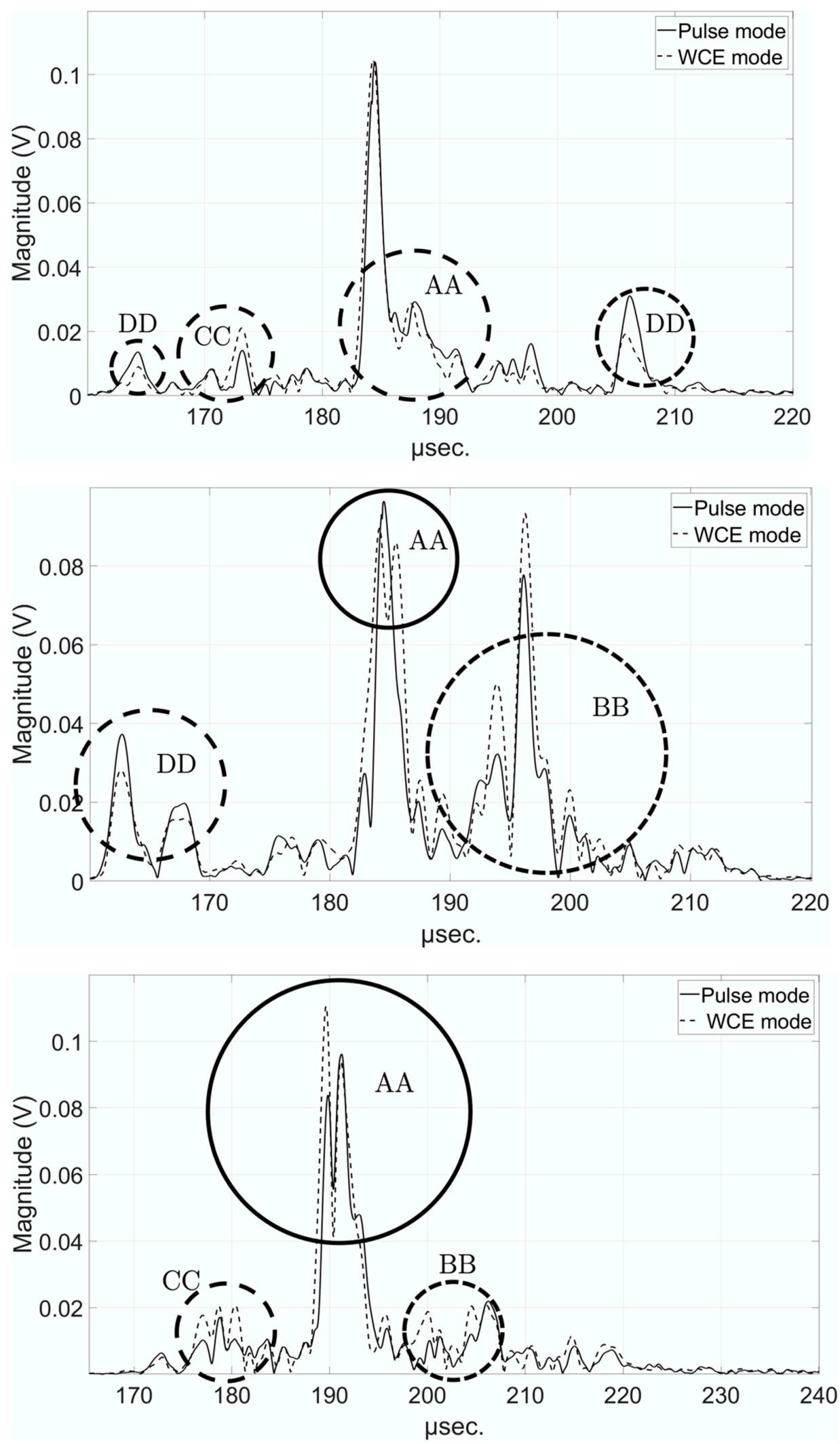

Figure 15. Detailed examples of the signals (envelope) at $1 \mathrm{MHz}$ for the chicken drumstick in pulse-mode (solid line) and in WCE-mode (dotted line) for the angles $5^{\circ}$ (top), $90^{\circ}$ (middle), and $336^{\circ}$ (bottom). AA: increase in resolution; BB: increase in amplitude and resolution; CC: increase in amplitude; DD: decrease in amplitude. 
At $1 \mathrm{MHz}$, the bandwidths in pulse-mode and in WCE-mode were identical, and equal to $900 \mathrm{kHz}$ for similar values of (0.5-1.4) MHz. At $2.25 \mathrm{MHz}$, the bandwidth increased between pulse-mode and WCE-mode, from $1 \mathrm{MHz}$ (pulse) to $1.8 \mathrm{MHz}$ (WCE); that is, about twice as much, which was remarkable. This corresponded well with the observation of the resolution of USCT. The multifrequency mode was strongly favored by the effect of high frequencies for a bandwidth up to almost $3 \mathrm{MHz}$. However, the bandwidth alone did not explain the differences between the two imaging modes. The study of the area under the curve (AUC) was interesting.

The AUC represents a spectral density and a quantity of energy propagated frequency by frequency. The higher the AUC, the more energy is propagated for each frequency in the bandwidth of the spectrum. At $500 \mathrm{kHz}$, the AUC in pulse-mode was higher than the AUC in WCE-mode, but the difference was not significant (12\%). For the two other frequencies, $1 \mathrm{MHz}$ and $2.25 \mathrm{MHz}$, the AUC was markedly higher in WCE-mode than in pulse-mode, with differences of $43 \%$ and $87 \%$, respectively. This strong increase reflected a better energy transport for the WCE-mode method, and thus explained the improvement of the CNR. The differences between the pseudo-wavelets and the theoretical analysis wavelets, which were not very significant $(<3 \%)$, did not influence this improvement. Despite the difficulty in experimentally "designing" a pseudo-wavelet such as a theoretical analyzing wavelet, the WCE-mode USCT remained more interesting.

\subsection{Intensity, Ultrasonic Field, and Beam Aperture}

One of the objectives of this work was to study the behavior of USCT when the signals were of low amplitudes, generating low-intensity waves and low pressures. The main idea was to limit the ultrasonic intensities so that USCT could be operated on children's fragile tissues. In B-mode ultrasound imaging, the intensity was between 30 and $200 \mathrm{~mW} / \mathrm{cm}^{2}$, and the time of exposure of the organ was a few seconds. In USCT, the acquisition time was longer (today USCT is not a real-time modality like B-mode ultrasound imaging) and the intensity needed to be as low as possible. In this study, the electro-acoustic devices were set to limit the spatial-peak temporal-average intensity $\left(\boldsymbol{I}_{\boldsymbol{s p t a}}\right)$ to values below $30 \mathrm{~mW} / \mathrm{cm}^{2}$. At $500 \mathrm{kHz}$, the measured $\boldsymbol{I}_{\text {spta }}$ was $27.6 \mathrm{~mW} / \mathrm{cm}^{2}$ (Table 5) for an $\mathrm{MI}=0.93$. These values were higher than those obtained with the transducers at $1 \mathrm{MHz}$ and $2.25 \mathrm{MHz}$, respectively $\left(\boldsymbol{I}_{\text {spta }}=18.8 \mathrm{~mW} / \mathrm{cm}^{2}(\mathrm{MI}=0.56)\right.$ and $\left.\boldsymbol{I}_{\text {spta }}=19.6 \mathrm{~mW} / \mathrm{cm}^{2}(\mathrm{MI}=0.38)\right)$. For all three frequencies, the MI values were well within expectations for pediatric use. The $I_{\text {spta }}$ were well below $30 \mathrm{~mW} / \mathrm{cm}^{2}$. The objective was achieved because, despite these low intensities, the resolution of USCT is satisfactory. The higher intensity at $500 \mathrm{kHz}$ compared with other frequencies explained the good results obtained with USCT at this low frequency, regardless of the pulse- or WCE-mode, because more energy was transported. The resolution decreased but the penetration increased, and combined with a large acoustic aperture of the beam, USCT had a correct CNR, strongly enhanced in WCE-mode. The lateral opening of the beam was about $40 \mathrm{~mm}$, and was $30 \mathrm{~mm}$ for the axial opening. This represented an insonification surface of about $12 \mathrm{~cm}^{2}$. For the newborn arm phantom, this surface was of the same order of magnitude as the diameter of the soft body. All areas of the object, bones and vessels, were sufficiently illuminated to return relevant information, even at low intensity and low acoustic impedance contrasts. The shape of the beam with a cylindrical aperture, for a small slice thickness and a large lateral aperture, favored complete organ insonification. This was particularly interesting in the case of the chicken drumstick, since this part was larger than the beam width. The disadvantage was that the object was required to be as central as possible. This was difficult to achieve, even with a precision scanner. This explained why part of the skin of the chicken drumstick (on the right of point 6) was less visible.

\subsection{Usefulness on Living Tissue}

In clinical application, and to strive for a fast and benign modality, it is not possible to use complex modeling for ultrasonic imaging of a complete organ containing skin, muscle, 
fat, and bone. The model must be simple and must assume that all propagation media are fluid media in which compressional waves propagate only at the velocity of a wave in water. Solutions that involve estimating the velocity distribution for the forward model (e.g., the studies by Renaud et al. [40] or Wiskin et al. [16]) are noteworthy, but are not adaptable to clinical practice today.

As shown in this study of an artificial phantom of a newborn arm and a nonliving chicken drumstick, the method made morphometric analysis possible. However, in this work, the quantity of error, caused by the fact that the sections demonstrated for $\mu \mathrm{CT}$ and USCT were not acquired exactly in the same plane, could not be estimated accurately. The thickness of the $\mu \mathrm{CT}$ section was $30 \mu \mathrm{m}$ and $50 \mu \mathrm{m}$, whereas the thickness of the USCT section was $3 \mathrm{~mm}$. Therefore, there was a distance between these two planes in the experiment. The acoustic signals, resulting from a superposition of high amplitude signals from the bone cortex with those interfering with the soft tissue, corresponded to a $3 \mathrm{D}$ integration of the field in the azimuthal plane. This was a limitation in determining the uncertainty of the morphometric analysis that should be investigated on multiple $\mu \mathrm{CT}$ and USCT slices. For a living arm or leg, the presence of physiological artifacts, such as tissue elasticity, should be taken into account to analyze the robustness of the method.

\section{Conclusions}

This work was focused on reflection-mode USCT of complete artificial and natural organs, and on the comparison of two ultrasonic wave transmission configurations, one based on a pulse and the other based on a wavelet mathematically adapted in frequency and time to the transducer. In the case of the latter, the contrast-to-noise ratio was improved. Three frequencies were analyzed and combined. Finally, the ultrasonic wave amplitude was controlled to meet pediatric clinical examination criteria, in terms of spatial-peak temporal-average intensity and mechanical index.

The organs analyzed were a phantom mimicking a newborn's arm containing blood vessels and single and joined bones, and an ex vivo chicken drumstick complete with skin, muscles, nerves, and two bones, tibia and fibula. The phantom was primarily intended for medical training purposes rather than research applications, and as a result, the bone parts did not reproduce realistic properties. By comparing USCT with $\mu \mathrm{CT}$ as a reference, organ morphometry was identifiable and measurable. The phantom blood vessels were differentiated, and so were the different muscles in the chicken drumstick. Even if the object was small, such as the fibula in the chicken drumstick, it could be detected by USCT. By modifying the transmitted signal to obtain a wavelet with mathematical properties, and by adapting the filter parameters during the inversion, reflection-mode USCT was very interesting. The gain of the CNR could be greatly increased at any frequency between $200 \mathrm{kHz}$ and $3 \mathrm{MHz}$. The image presented artifacts due to different uncontrolled physical processes (multiple echoes and scattering, strong variation of propagation velocity, and attenuation), but the dimensions of the soft tissue and bone contours, even if they were small, such as for the fibula of the chicken drumstick, were satisfactory to differentiate between different regions of interest. The reflection-mode USCT based on wavelet processing allowed high-contrast anatomical and morphometric imaging and, even at low intensity, allowed highly accurate measurement of interdiaphyseal distances, for example, or of the volume of a fibrous muscle. The work objective of an improved visualization of the various organs by reflection-mode USCT was reached, and this modality, in its fast and low-intensity version, can be a qualitative and morphometric tissue imaging modality, namely in clinical pediatrics.

Author Contributions: Conceptualization, P.L.; methodology, E.D. and P.L.; software, E.D. and P.L.; validation, E.D. and P.L.; formal analysis, E.D., L.S., V.L., and P.L.; investigation, E.D., L.S., V.L., and P.L.; resources, L.S. and P.L.; data curation, L.S. and P.L.; writing-original draft preparation, E.D. and P.L.; writing-review and editing, P.L.; visualization, P.L.; supervision, P.L.; project administration, P.L.; funding acquisition, P.L. All authors have read and agreed to the published version of the manuscript. 
Funding: This research was funded by the Initiative d'Excellence of the Aix Marseille UniversityA*MIDEX, a French Investissements d'Avenir program (no 004343). It was carried out within the framework of the Mechanics and Engineering Institute, Aix Marseille University. Elise Doveri's doctoral thesis was funded by the French Ministry of Research.

Institutional Review Board Statement: No ethical review or approval was required in this study. The chicken drumstick was purchased at a food store and could be used without specific restrictions.

Acknowledgments: The authors are grateful to Mélanie Brie from Aix-Marseille University and Jeanne Baldisser from Centrale Marseille for their internship work. The authors are grateful to Mustafa Ali Misri, business manager; Faiqa Khzir, operations officer; and Adrian Wydra, chief technology officer of True Phantom Solutions in Windsor, Canada, for their assistance in the fabrication of the newborn arm phantom and the evaluation of some properties of the materials used.

Conflicts of Interest: The authors declare no conflict of interest. The funders had no role in the design of the study; in the collection, analysis, or interpretation of the data; in the writing of the manuscript; or in the decision to publish the results.

\section{References}

1. Griffith, J.F. Diagnostic Ultrasound: Musculoskeletal, 2nd ed.; Amirsys Publishing: Salt Lake City, UT, USA, 2019; ISBN 978-1-937242-17-6.

2. Riccabona, M. (Ed.) Pediatric Ultrasound: Requisites and Applications, 2nd ed.; Springer: Cham, Switzerland, 2020; ISBN 978-3-030-47909-1.

3. Aldrich, J.E. Basic physics of ultrasound imaging. Crit. Care Med. 2007, 35, S131-S137. [CrossRef] [PubMed]

4. André, M.P.; Martin, P.J.; Otto, G.P.; Olson, L.K.; Barrett, T.K.; Spivey, B.A.; Palmer, D.A. A New Consideration of Diffraction Computed Tomography for Breast Imaging: Studies in Phantoms and Patients. In Acoustical Imaging; Jones, J.P., Ed.; Springer: Boston, MA, USA, 1995; pp. 379-390, ISBN 978-1-4615-1943-0.

5. Mensah, S.; Ferriere, R. Diffraction tomography: A geometrical distortion free procedure. Ultrasonics 2004, 42, 677-682. [CrossRef] [PubMed]

6. Lefebvre, J.-P.; Lasaygues, P.; Mensah, S. Acoustic Tomography, Ultrasonic Tomography. In Materials and Acoustics Handbook; Bruneau, M., Potel, C., Eds.; ISTE: London, UK, 2009; pp. 887-906, ISBN 978-0-470-61160-9.

7. Hopp, T.; Ruiter, N.; Bamber, J.C.; Duric, N.; Van Dongen, K.W.A. Proceedings of the International Workshop on Medical Ultrasound Tomography, Speyer, Germany, 1-3 November 2017; KIT Scientific Publishing: Karlruhe, Germany, 2017.

8. Lasaygues, P.; Guillermin, R.; Lefebvre, J.P. Ultrasonic Computed Tomography. In Bone Quantitative Ultrasound; Springer: Berlin/Heidelberg, Germany, 2011; pp. 441-459.

9. Wang, K.; Matthews, T.; Anis, F.; Li, C.; Duric, N.; Anastasio, M.A. Breast Ultrasound Computed Tomography Using Waveform Inversion with Source Encoding. In Proceedings of the Medical Imaging 2015: Ultrasonic Imaging and Tomography; Bosch, J.G., Duric, N., Eds.; SPIE: Orlando, FL, USA, 2015; p. 94190C.

10. Sandhu, G.Y.; Li, C.; Roy, O.; Schmidt, S.; Duric, N. Frequency domain ultrasound waveform tomography: Breast imaging using a ring transducer. Phys. Med. Biol. 2015, 60, 5381-5398. [CrossRef] [PubMed]

11. Bernard, S.; Monteiller, V.; Komatitsch, D.; Lasaygues, P. Ultrasonic computed tomography based on full-waveform inversion for bone quantitative imaging. Phys. Med. Biol. 2017, 62, 7011-7035. [CrossRef] [PubMed]

12. Pérez-Liva, M.; Herraiz, J.L.; Udías, J.M.; Miller, E.; Cox, B.T.; Treeby, B.E. Time domain reconstruction of sound speed and attenuation in ultrasound computed tomography using full wave inversiona. J. Acoust. Soc. Am. 2017, 141, 1595-1604. [CrossRef] [PubMed]

13. Calderon Agudo, O.; Guasch, L.; Huthwaite, P.; Warner, M. 3D Imaging of the Breast Using Full-Waveform Inversion; Bamber, J., van Dongen, K.W.A., Duric, N., Hopp, T., Ruiter, N.V., Eds.; KIT Scientific Publishing: Karlruhe, Germany, 2017; pp. 99-110.

14. Falardeau, T.; Belanger, P. Ultrasound tomography in bone mimicking phantoms: Simulations and experiments. J. Acoust. Soc. Am. 2018, 144, 2937-2946. [CrossRef] [PubMed]

15. Wiskin, J.W.; Malik, B.; Natesan, R.; Pirshafiey, N.; Klock, J.; Lenox, M. 3D full inverse scattering ultrasound tomography of the human knee (Conference Presentation). In Proceedings of the Medical Imaging 2019: Ultrasonic Imaging and Tomography; Ruiter, N.V., Byram, B.C., Eds.; SPIE: San Diego, CA, USA, 2019; p. 25.

16. Wiskin, J.; Malik, B.; Borup, D.; Pirshafiey, N.; Klock, J. Full wave 3D inverse scattering transmission ultrasound tomography in the presence of high contrast. Sci. Rep. 2020, 10, 20166. [CrossRef] [PubMed]

17. Guasch, L.; Calderón Agudo, O.; Tang, M.-X.; Nachev, P.; Warner, M. Full-waveform inversion imaging of the human brain. NPJ Digit. Med. 2020, 3, 28. [CrossRef] [PubMed]

18. Espinosa, L.; Doveri, E.; Bernard, S.; Monteiller, V.; Guillermin, R.; Lasaygues, P. Ultrasonic Imaging of High-contrasted Objects Based on Full-waveform Inversion: Limits under Fluid Modeling. Ultrason. Imaging 2021, 43, 88-99. [CrossRef] [PubMed]

19. Lefebvre, J.-P. Progress in linear inverse scattering imaging: NDE application of Ultrasonic Reflection Tomography. In Inverse Problem in Engineering Mechanics; Balkema, A.A., Ed.; Brookfield: Rotterdam, The Netherlands, 1994; pp. 371-375. 
20. Zheng, R.; Lasaygues, P. Simultaneous Assessment of Bone Thickness and Velocity for Ultrasonic Computed Tomography Using Transmission-Echo Method. In Proceedings of the 2013 IEEE International Ultrasonics Symposium (IUS), Prague, Czech Republic, 21-25 July 2013; pp. 2084-2087.

21. Shortell, M.P.; Althomali, M.A.M.; Wille, M.-L.; Langton, C.M. Combining Ultrasound Pulse-Echo and Transmission Computed Tomography for Quantitative Imaging the Cortical Shell of Long-Bone Replicas. Front. Mater. 2017, 4, 40. [CrossRef]

22. Lasaygues, P. Assessing the cortical thickness of long bone shafts in children, using two-dimensional ultrasonic diffraction tomography. Ultrasound Med. Biol. 2006, 32, 1215-1227. [CrossRef] [PubMed]

23. Duric, N.; Littrup, P.; Poulo, L.; Babkin, A.; Pevzner, R.; Holsapple, E.; Rama, O.; Glide, C. Detection of breast cancer with ultrasound tomography: First results with the Computed Ultrasound Risk Evaluation (CURE) prototype: Detection of breast cancer with ultrasound tomography. Med. Phys. 2007, 34, 773-785. [CrossRef] [PubMed]

24. Rouyer, J.; Mensah, S.; Vasseur, C.; Lasaygues, P. The benefits of compression methods in acoustic coherence tomography. Ultrason. Imaging 2015, 37, 205-223. [CrossRef] [PubMed]

25. Lasaygues, P.; Arciniegas, A.; Espinosa, L.; Prieto, F.; Brancheriau, L. Accuracy of coded excitation methods for measuring the time of flight: Application to ultrasonic characterization of wood samples. Ultrasonics 2018, 89, 178-186. [CrossRef] [PubMed]

26. Loosvelt, M.; Lasaygues, P. A Wavelet-Based Processing method for simultaneously determining ultrasonic velocity and material thickness. Ultrasonics 2011, 51, 325-339. [CrossRef] [PubMed]

27. Metwally, K.; Lefevre, E.; Baron, C.; Zheng, R.; Pithioux, M.; Lasaygues, P. Measuring mass density and ultrasonic wave velocity: A wavelet-based method applied in ultrasonic reflection mode. Ultrasonics 2016, 65, 10-17. [CrossRef] [PubMed]

28. Lasaygues, P.; Guillermin, R.; Metwally, K.; Fernandez, S.; Balasse, L.; Petit, P.; Baron, C. Contrast resolution enhancement of Ultrasonic Computed Tomography using a wavelet-based method-Preliminary results in bone imaging. In Proceedings of the International Workshop on Medical Ultrasound Tomography; Bamber, J., van Dongen, K.W.A., Duric, N., Hopp, T., Ruiter, N.V., Eds.; KIT Scientific Publishing: Karlruhe, Germany, 2017; pp. 291-302.

29. Retz, K.; Kotopoulis, S.; Kiserud, T.; Matre, K.; Eide, G.E.; Sande, R. Measured acoustic intensities for clinical diagnostic ultrasound transducers and correlation with thermal index: Acoustic intensity and TI. Ultrasound Obstet. Gynecol. 2017, 50, 236-241. [CrossRef] [PubMed]

30. Preston, R.C. Output Measurements for Medical Ultrasound.; Springer: London, UK, 1991; ISBN 978-1-4471-1883-1.

31. Kak, A.; Slaney, M. Principles of Computerized Tomographic Imaging; Society for Industrial and Applied Mathematics: Philadelphia, PA, USA, 2001.

32. Deans, S.R. The Radon Transform and some of Its Applications; Dover Publications: Mineola, NY, USA, 2007; ISBN 978-0-486-46241-7.

33. Devaney, A.J. Mathematical Foundations of Imaging, Tomography and Wavefield Inversion; Cambridge University Press: Cambridge, MA, USA, 2012; ISBN 978-0-521-11974-0.

34. Holschneider, M. Inverse Radon transforms through inverse wavelet transforms. Inverse Probl. 1991, 7, 853-861. [CrossRef]

35. Jaffard, S. Construction of Wavelets on Open Sets. In Wavelets; Combes, J.-M., Grossmann, A., Tchamitchian, P., Eds.; Springer: Berlin/Heidelberg, Germany, 1989; pp. 247-252, ISBN 978-3-642-97179-2.

36. Jaffard, S.; Meyer, Y.; Ryan, R.D. Wavelets: Tools for Science and Technology; Society for Industrial and Applied Mathematics: Philadelphia, PA, USA, 2001; ISBN 978-0-89871-448-7.

37. Meyer, Y. Orthonormal Wavelets. In Wavelets; Combes, J.-M., Grossmann, A., Tchamitchian, P., Eds.; Springer: Berlin/Heidelberg, Germany, 1989; pp. 21-37, ISBN 978-3-642-97179-2.

38. Schindelin, J.; Arganda-Carreras, I.; Frise, E.; Kaynig, V.; Longair, M.; Pietzsch, T.; Preibisch, S.; Rueden, C.; Saalfeld, S.; Schmid, B.; et al. Fiji: An open-source platform for biological-image analysis. Nat. Methods 2012, 9, 676-682. [CrossRef]

39. National Council on Radiation Protection and Measurements (Ed.) Biological Effects of Ultrasound: Mechanisms and Clinical Implications; NCRP report; The Council: Bethesda, MD, USA, 1983; ISBN 978-0-913392-64-5.

40. Renaud, G.; Kruizinga, P.; Cassereau, D.; Laugier, P. In vivo ultrasound imaging of the bone cortex. Phys. Med. Biol. 2018, 63, 125010. [CrossRef] [PubMed] 